\title{
Magnetohydrodynamic Density Waves in a Composite Disk System of Interstellar Medium and Cosmic-Ray Gas
}

\author{
YU-QING LOU ${ }^{1,2,3}$ AND ZuhuI FAN ${ }^{4}$
}

${ }^{1}$ National Astronomical Observatories Chinese Academy of Sciences A20 Datun Road, ChaoYang District, Beijing, 100012, China

${ }^{2}$ Physics Department, Tsinghua Astrophysics Center, Tsinghua University, Beijing, 100084, China

${ }^{3}$ Department of Astronomy and Astrophysics The University of Chicago Chicago, Illinois 60637, USA lou@oddjob.uchicago.edu

${ }^{4}$ Beijing Astrophysical Center and Department of Astronomy, CAS-PKU, Beijing, 100871, China 


\begin{abstract}
Multi-wavelength observations from radio to soft X-ray bands of large-scale galactic spiral structures offer synthesized and comprehensive views of nearby disk galaxies. In the presence of a massive dark-matter halo, the density-wave dynamics on galactic scales involves the stellar disk, the gas disk of interstellar medium (ISM), the magnetic field, and the cosmic-ray gas (CRG). In this paper, we explore the dynamic and electromagnetic interplay between the magnetized ISM disk and CRG disk so that structural and diagnostic features of optical, infrared, and synchrotron radio-continuum emissions from a spiral galaxy can be physically understood. On timescales of galactic density waves, cosmic rays collectively may be treated as a relativistically hot tenuous gas fluid that is tied to the large-scale mean magnetic field in transverse bulk motions but moves otherwise differently along the magnetic field relative to the ISM. For both fast and slow magnetohydrodynamic (MHD) density waves in a composite disk system of magnetized ISM and CRG, the minute CRG mass density enhancement is phase shifted relative to the enhancement of parallel magnetic field. Owing to an extremely small number of cosmic rays, the large-scale magnetic field enhancement dominates in synchrotron radio-continuum emissions (as if the CRG is almost unperturbed) for spiral structural manifestations. In addition to the fast and slow MHD density waves, there also exists a suprathermal MHD wave mode by which CRG adjusts itself with an effective suprathermal sound speed close to the speed of light $c$.
\end{abstract}

Key words: galaxies: cosmic rays - density waves — radio polarization - gravitation — magnetic fields — interstellar medium 


\section{INTRODUCTION}

The entire system of a spiral galaxy consists of a massive dark-matter halo, an older stellar halo, a luminous stellar disk with an central ellipsoidal bulge, a thin disk of interstellar medium (ISM) consisting of a thermal gas plus dusts, relativistic cosmic rays, and a large-scale magnetic field (e.g., Woltjer 1965). The rotation curve and speed of a disk galaxy are dominantly controlled by the total mass (dark matter included) distribution in the system via gravity (e.g., Kormendy \& Norman 1979; Kent 1986, 1987, 1988). The spiral structure in the disk seen in red light from the relatively old stellar population is usually broad and smooth (e.g. Elmegreen 1981), while the spiral arm structure in the disk seen in blue light from the young stellar population is typically brilliant, narrow, and sharp. Often, there exist dark narrow dust lanes lying along inner edges of the luminous spiral arms outlined by young O, B stars and HII complexes. The large-scale spiral structure seen in total synchrotron radio-continuum emissions well tracks the optical spiral structure (e.g., the "Whirlpool galaxy" M51 [NGC 5194]; Mathewson, van der Kruit, \& Brouw 1972; Neininger 1992; Berkhuijsen et al. 1997). Polarized radiocontinuum emission arms also follow optical spiral arms as seen in several nearby spiral galaxies (e.g. M51, M31, NGC 2997). However, the spiral structure seen in polarized synchrotron radio-continuum emissions can, in the case of the nearby spiral galaxy NGC 6946 (Beck \& Hoernes 1996; Fan \& Lou 1996, 1999; Lou \& Fan 1998a, 2002; Ferguson et al. 1998; Frick et al. 2000; Lou 2002), appear interlaced with the optical spiral structure. ${ }^{1}$ Synchrotron radio-continuum emissions are produced by relativistic cosmic-ray electrons gyrating around magnetic fields. The large-scale interrelations between optical and radio-continuum spiral structures seen in spiral galaxies hint at an intricate magnetohydrodynamic (MHD) coupling among various seemingly unrelated processes on distinctly different scales (Lou \& Fan 1997, 1998b, 2000a, b; Lou 2002).

In addition to giving rise to a more or less flat disk rotation curve, the massive dark-matter halo also helps prevent rapid development of bar-type instabilities that have been shown to exist through numerical and theoretical studies (Miller, Prendergast, \& Quirk 1970; Hohl 1971; Ostriker \& Peebles 1973; Bardeen 1975; Shu et al. 2000). By joint effects of differential rotation, epicyclic oscillations, and self-gravity, such a disk system is still vulnerable to various instabilities (Safronov 1960; Toomre 1964; Goldreich \& Lynden-Bell 1965; Julian \& Toomre 1966; Jog \& Solomon 1984a, b; Binney \& Tremaine 1987; Bertin et al. 1989a, b; Bertin \& Lin 1996; Montenegro et al. 1999; Lou, Yuan, \& Fan 2001). In the case of a stellar disk, such instabilities may ultimately lead to an effective increase of stellar velocity dispersion. In the case of a rotating gas disk, such instabilities may trigger cloud and star formations on global scales (Quirk 1972; Jog \& Solomon 1984a, b; Kennicutt 1989; Kennicutt et al. 1994; Wang \& Silk 1994; Elmegreen 1995; Jog 1992, 1996; Silk 1997; Lou \& Fan 1998b). As clouds and stars directly

1 Two other late-type gas-rich spiral galaxies nearby, IC342 (Krause 1993; Crosthwatte et al. 2000) and M83 or NGC 5236 (Sukumar \& Allen 1989; Neininger, Beck, Sukumar, \& Allen 1993), also bear somewhat similar interlaced arm features of NGC 6946. 
form in the gas disk of interstellar medium (ISM) and the magnetic and thermal energy densities in the ISM are roughly comparable, magnetic field should play an important role in affecting the global star formation rate. In particular, magnetic field introduces additional MHD instabilities (Lou \& Fan 1997, 1998a, 2000a, b; Lou et al. 2001a) that are independent of Toomre-type ring instabilities for axisymmetric disturbances and that can lead to nonaxisymmetric gravitational collapses (Lou 1996a; Lou et al. 2001a).

Over the past decade, the advent of Infrared Astronomical Satellite (IRAS) and Infrared Space Observatory (ISO) has opened up the infrared window for systematic, extensive, and unprecedented galactic observations. One important discovery of IRAS was the remarkably tight global correlation between integrated farinfrared and radio-continuum emissions from spiral galaxies (Dickey \& Salpeter 1984; Helou, Soifer \& Rowan-Robinson 1985; de Jong et al. 1985; Wunderlich, Klein \& Wielebinski 1987; Bicay \& Helou 1990; Helou 1991). This empirical fact implies a close physical connection between two apparently unrelated physical mechanisms, namely, thermal emissions from UV photon heated dusts in the ISM and synchrotron radio-continuum emissions from relativistic cosmic-ray electrons gyrating around galactic magnetic field. It was proposed that massive star formation in the ISM is likely the key process that somehow links these disparate radiative phenomena (Harwit \& Pacini 1975; Helou et al. 1985; Condon, Anderson, \& Helou 1991). Recent high-resolution ISO observations of the nearby spiral galaxes NGC 6946 (e.g. Tuffs et al. 1996; Lu et al. 1996; Helou et al. 1996; Malhotra et al. 1996) and M31 (see extensive references in Berkhuijsen, Beck \& Walterbos 2000) further revealed detailed spatial correlations and correspondences between large-scale infrared and radio structures as already partially evidenced in an earlier IRAS survey of spiral galaxies (Bicay \& Helou 1990). We emphasize the importance of multi-wavelength observations of spiral galaxies (Lou, Walsh, Han \& Fan 2002), because we believe that large-scale spiral MHD density waves are the underlying dynamic process that links and organizes the various ISM components through magnetic field and that gives rise to large-scale interrelations of spiral structures revealed in various wavebands (Lou \& Fan 2000a, b).

The familiar hydrodynamic density wave theory (Lin \& Shu 1964, 1966) dealt primarily with large-scale perturbations in a thin stellar disk, using either a formalism of stellar distribution function (e.g., Toomre 1964; Julian \& Toomre 1966) or a fluid approach (e.g., Lin 1967b, 1987; Toomre 1977; Binney \& Tremaine 1987; Bertin \& Lin 1996). The inclusion of a less massive ISM disk was, in the past, regarded as a passive effect. Nevertheless, there has been growing evidence for the important dynamic as well as diagnostic roles of the ISM (e.g., Jog \& Solomon $1984 \mathrm{a}, \mathrm{b})$. While smaller than the kinetic energy density of the galactic disk rotation, the energy densities of thermal ISM, magnetic field, and cosmic rays are comparable (e.g., Lin 1967a). The increase of dynamical freedoms in the magnetized ISM may lead to different large-scale structural interrelations in multi-wavelength observations and the presence of magnetic field would significantly influence the global star formation rate in a nontrivial manner. Therefore, in order to understand large-scale infrared and synchrotron radio-continuum structures of spiral galaxies as well as their interrelations with the optical spiral structures, it becomes necessary to take into account the effects of thermal ISM, magnetic field, and cosmic rays within the overall MHD density wave scenario. While the gravita- 
tional effect dominates the dynamics of the massive stellar disk and of the mutual interaction between the stellar and ISM disks (Lou \& Fan 1997, 1998b, 2000a, b), physical consequences of the dynamical interaction among the sub-systems remain to be specifically and thoroughly examined.

While the ultimate goal of building up such an edifice remains challenging, it is nonetheless physically informative to extract necessary ingredients separately from such a comprehensive scenario in order to work out simpler yet non-trivial physical partial problems in concrete terms. This research strategy requires a systematic investigation on a series of partial problems involving various simplifications. Meanwhile, one must clearly bear in mind the utility as well as limitations of the theoretical results thus derived. We hope to gain physical insights for the overall problem such that various aspects can be modeled quantitatively to confront multi-wavelength observations of spiral galaxies (Lou et al. 2002).

We have shown in earlier publications the possible existence of fast and slow MHD density waves $^{2}$ in a differentially rotating thin gas disk embedded with an azimuthal magnetic field (Fan \& Lou 1996; Lou \& Fan 1998a). In particular, perturbation enhancements of thermal gas density and parallel magnetic field are roughly in phase for fast MHD density waves but are significantly phase shifted for slow MHD density waves with a phase difference $\gtrsim \pi / 2$. The features of fast MHD density waves may explain why optical and synchrotron radio-continuum structures nearly coincide in several nearby spiral galaxies (e.g. M51, M31, NGC 2997 etc.), whereas the features of slow MHD density waves may explain why magnetic spiral arms lie in between the optical spiral arms in the galaxy NGC 6946 and why such an interlaced spiral structure persists over a large spatial scale (Beck \& Hoernes 1996; Fan \& Lou 1996; Ferguson et al. 1998; Frick et al. 2000; Lou \& Fan 1998a, 2002).

An important new realm of applications for spiral MHD density wave theory has been developed recently for circumnuclear spiral arms and starburst "rings" on kiloparsec scales in the central regions around nuclei of disk galaxies such as NGC 1097, NGC 6951, NGC 2997, and NGC 2207 etc. (Lou, Yuan, Fan, \& Leon 2001). More specifically, when coupled with proper wave damping mechanisms in a circumnuclear disk, spiral MHD density waves play the key role of persistently removing angular momentum from the central magnetized gas disk in the circumnuclear region, and the incessant accumulation of gas materials and magnetic flux in a roughly circular zone is eventually vulnerable to gravitational instabilities (e.g., Elmegreen 1994; Lou 1996a) and thus leads to the appearance of a starburst "ring" somewhere inside the modified inner Lindblad resonance (Lou et al. 2001b). At the current stage of observations, wavelet analyses and reconstructions on high-resolution optical images from Hubble Space Telescope (HST) have revealed circumnuclear spiral structures with or without nuclear bars in several dozens of spiral galaxies, including NGC 2207 (Yuan, private communications 2001). The starburst galaxy M83 (NGC 5236) has been revealed to possess double circumnuclear ring and minibar on scales of several hundred parsecs (Elmegreen

${ }^{2}$ Note that incompressible Alfvénic fluctuations perpendicular to a magnetized rotating gas disk may exist (sort of bending MHD waves), but they do not directly couple to gravitational potential perturbations in the absence of mass density fluctuations (see the formulation contained in $\S 2$ and in Appendix C). 
et al. 1998). In comparison, preliminary information of circumnuclear magnetic fields are only known recently for NGC 1097 (Beck et al. 1999) and barely for NGC 2997 (Han et al. 1999; Lou et al. 1999). Here again, it is the relativistic cosmic-ray electrons abundant in the circumnuclear environs that reveal valuable clues of trailing swirl patterns of circumnuclear magnetic fields.

The central theme of this paper is to investigate the coupling of spiral MHD density waves in the magnetized thermal ISM disk and the cosmic-ray gas (CRG). We are mainly motivated by the fact that the energy densities of thermal gas, magnetic field, and CRG are comparable (all on the order of $\sim 10^{-12} \mathrm{erg} \mathrm{cm}^{-3}$ ), the fact that galactic synchrotron radio-continuum emissions result from interactions of relativistic cosmic-ray electrons with the magnetic field, and the fact that magnetic field appears to be the underlying cause for the large-scale structural correlations as revealed by multi-wavelength observations of nearby spiral galaxies. We do not include the effect of the massive stellar disk in the formulation merely for the sake of simplicity; yet we have enough confidence, based on our earlier analyses (Lou \& Fan 1997, 1998b, 2000a, b), that the gross properties of fast and slow MHD density waves should remain in the magnetized thermal gas disk of ISM even when the more massive stellar disk is included (see Lou et al. 2001a; Lou \& Shen 2003).

Our basic theoretical formalism of the interaction between the magnetized thermal gas and CRG closely follows that pioneered by Parker (1965, 1967, 1969) nearly four decades ago. The key starting point is to treat galactic cosmic rays collectively as a tenuous relativistically hot gas fluid for dynamic phenomena of large spatial scales (i.e. $\gg$ gyroradii of cosmic rays) and low frequencies. In this paper, we therefore ignore resistive effects such that magnetic field is frozen into both ISM and CRG. The physical meaning of frozen-in condition is that bulk materials cannot go across magnetic field lines but are allowed to move along magnetic field lines. As ISM and CRG are two qualitatively different types of conducting fluids, their bulk motions along magnetic field lines are different following the proper dynamics. In other words, both thermal ISM and CRG are tied to the galactic magnetic field in transverse bulk motions but are allowed to move relative to each other along magnetic field lines. By this analysis, it is then possible to assess the relative importance of the enhancements of parallel magnetic field and CRG mass density in synchrotron radio-continuum structures of spiral galaxies.

The plan of the paper is as follows. Physical considerations and perturbation formulation of the problem are developed in $\S 2$. Dispersion relations of fast and slow MHD density waves and of the suprathermal MHD mode are derived in $\S 3$ using the tight-winding approximation. Galactic applications are described in $\S 4$. Notes and discussions are contained in $\S 5$. Appendix A contains some mathematical details of the key perturbation equation (3.12) for the convenience of reference and discussion. In Appendix B, we derive and summarize specific phase relationships of perturbation variables for the problem studied by Parker (1965) yet with the displacement current effect included. In Appendix C, we provide a first-principle formulation as the very basis of our MHD perturbation analysis. 


\section{FORMULATION OF THE PROBLEM}

For the mathematical description of the problem under consideration (see Appendix $\mathrm{C}$ for more specifics), we adopt the cylindrical coordinate system $(r, \theta, z)$ with the $z$ - and disk rotation axes being coincident. For the stationary rotational equilibrium of the background, we presume the bulk azimuthal speed $U_{\theta}$ of the CRG to be the same as the bulk azimuthal speed $V_{\theta}$ of the thermal gas of ISM. ${ }^{3}$ The background magnetic field $B_{\theta}$ is azimuthal to avoid the magnetic field winding dilemma due to the disk differential rotation (Lynden-Bell 1966; Roberts \& Yuan 1970). The profile of the background magneto-rotational equilibrium is characterized by $U_{\theta}=V_{\theta}=\Omega r$ and $B_{\theta}=F_{B} / r$, where $\Omega(r)$ is the angular rotation rate and $F_{B}$ is a constant. The stationary radial momentum equation for the background becomes

$$
-\frac{\left(\mu_{\circ}+\epsilon_{\circ}\right) V_{\theta}^{2}}{r}=-\frac{\partial\left(p_{\circ}+P_{\circ}\right)}{\partial r}-\int d z \frac{B_{\theta}}{4 \pi r} \frac{\partial\left(r B_{\theta}\right)}{\partial r}+\left(\mu_{\circ}+\epsilon_{\circ}\right) \frac{\partial \phi_{T}}{\partial r}
$$

(Lou 1996b; see Appendix C), where $\mu_{\circ}$ is the surface mass density of the thermal gas, $p_{\circ}$ is the two-dimensional thermal gas pressure, $\phi_{T}$ is the total negative gravitational potential, $\epsilon_{\circ} \equiv \Sigma_{\circ}+\Gamma P_{\circ} /\left[(\Gamma-1) c^{2}\right], \Sigma_{\circ}$ is the vertically integrated mass density of the CRG, $P_{\circ}$ is the two-dimensional effective CRG pressure taken to be isotropic (Parker 1969), $c$ is the speed of light, and $\Gamma$ is the polytropic index of the CRG (e.g., $\Gamma=4 / 3$ in a relativistically hot gas). The axisymmetric Poisson equation is

$$
\frac{1}{r} \frac{\partial}{\partial r}\left(r \frac{\partial \phi_{T}}{\partial r}\right)+\frac{\partial^{2} \phi_{T}}{\partial z^{2}}=-4 \pi \rho_{T},
$$

with $\phi_{T}$ felt at the magnetized gas disk being determined by the distribution of the total mass density $\rho_{T}$ in the entire system. Within the solar system, the CRG was observed to be remarkably isotropic with an upper bound on anisotropy of $\sim 10^{-3}$ (Greisen 1960). In general, small-scale plasma instabilities are expected to reduce the anisotropy of the CRG to be $\lesssim 1 \%$ (Lerche \& Parker 1966; Lerche 1967). Thermal gas pressure, effective CRG pressure, and magnetic energy density $B_{\theta}^{2} /(8 \pi)$ are roughly comparable and are on the order of $\sim 10^{-12} \mathrm{erg} \mathrm{cm}^{-3}$.

The thickness of the composite disk system is expediently taken to be infinitely thin here. In reality, the vertical quasi-static balance involves the confinement of the thermal ISM, CRG, and magnetic field under the action of gravity towards the galactic disk plane at $z=0$ from both sides. In the background rotational equilibirum, the radial Lorentz force in equation (2.1) vanishes (i.e., force-free) as $r B_{\theta}$ is taken to be a constant $F_{B}$. By this choice of $B_{\theta}$ that scales as $r^{-1}$, one needs to invoke some processes in the vicinity of the galactic centre to avoid the outright singularity of $B_{\theta}$ there. As the massive dark-matter halo contributes to $\rho_{T}$ and thus to $\phi_{T}$, the disk rotation curve for the velocity $V_{\theta S}(r)$ of the stellar disk is actually used to infer $\phi_{T}$ from observations. Since the thermal energy

${ }^{3}$ In general, $V_{\theta}$ and $U_{\theta}$ may be different. In galactic contexts, this difference should be small, because the total gravity (that of dark matter halo included) dominates over pressure and Lorentz forces. Furthermore, Kelvin-Helmholtz instabilities induced by small differences in $V_{\theta}$ and $U_{\theta}$ may sustain a certain level of microturbulence that tends to smooth out such velocity shear. 
density of the ISM is much smaller than the kinetic energy density of the galactic rotation and the magnetic field is taken to be force-free, the difference between the gas rotation velocity $V_{\theta}(r)$ and the stellar rotation velocity $V_{\theta S}(r)$ should be very small. In fact, one usually prescribes a $V_{\theta}(r)$ a priori based on observational input to construct an approximate background profile.

Given a background profile for the thin rotating gas disk of magnetized ISM together with the CRG, it is fairly straightforward to write down MHD equations for large-scale coplanar perturbations, that is, we limit the consideration to two-dimensional propagations of MHD wave perturbations tangential to the disk plane at $z=0$. By this requirement, incompressible Alfvénic fluctuations involving velocity and magnetic field perturbations perpendicular to the disk plane are excluded (Lou \& Fan 1998a). Likewise, Parker instability (Parker 1966) and magnetorotational instabilities (MRI; Balbus \& Hawley 1998; Kim \& Ostriker 2000) are also excluded. In the context of spiral MHD density waves, we are mainly interested in compressible fluctuations, for which the relevant MHD perturbation equations are given below explicitly. The radial component of the magnetic induction equation for the radial magnetic field perturbation $b_{r}$ is

$$
\frac{\partial b_{r}}{\partial t}=-\frac{1}{r} \frac{\partial\left(V_{\theta} b_{r}\right)}{\partial \theta}+\frac{1}{r} \frac{\partial\left(B_{\theta} v_{r}\right)}{\partial \theta},
$$

where $v_{r}$ is the bulk radial thermal gas velocity perturbation, and the azimuthal magnetic induction equation for the azimuthal magnetic field perturbation $b_{\theta}$ is

$$
\frac{\partial b_{\theta}}{\partial t}=\frac{\partial\left(V_{\theta} b_{r}\right)}{\partial r}-\frac{\partial\left(B_{\theta} v_{r}\right)}{\partial r}
$$

The divergence-free condition of the magnetic field perturbation $\vec{b} \equiv\left(b_{r}, b_{\theta}, 0\right)$ is

$$
\frac{1}{r} \frac{\partial\left(r b_{r}\right)}{\partial r}+\frac{1}{r} \frac{\partial b_{\theta}}{\partial \theta}=0
$$

While the bulk radial velocity $u_{r}$ of the CRG is the same as $v_{r}$ as restricted by the frozen-in magnetic field on large scales, the bulk azimuthal flow speed $u_{\theta}$ of the CRG and the bulk azimuthal flow speed $v_{\theta}$ of the thermal gas can however be different along magnetic field lines.

The radial component of the perturbed momentum equation is

$$
\begin{aligned}
\frac{\partial v_{r}}{\partial t} & -\frac{2 \mu_{\circ} V_{\theta} v_{\theta}}{r\left(\mu_{\circ}+\epsilon_{\circ}\right)}-\frac{2 \epsilon_{\circ} V_{\theta} u_{\theta}}{r\left(\mu_{\circ}+\epsilon_{\circ}\right)}+\frac{V_{\theta}}{r} \frac{\partial v_{r}}{\partial \theta}-\frac{(\mu+\epsilon)}{\left(\mu_{\circ}+\epsilon_{\circ}\right)^{2}} \frac{d\left(p_{\circ}+P_{\circ}\right)}{d r} \\
& =-\frac{1}{\left(\mu_{\circ}+\epsilon_{\circ}\right)} \frac{\partial(p+P)}{\partial r}-\int \frac{d z B_{\theta}}{4 \pi r\left(\mu_{\circ}+\epsilon_{\circ}\right)}\left[\frac{\partial\left(r b_{\theta}\right)}{\partial r}-\frac{\partial b_{r}}{\partial \theta}\right]+\frac{\partial \phi}{\partial r}
\end{aligned}
$$

(Lou 1996b; see Appendix C), where $\mu, \epsilon, p$, and $P$ are perturbations in $\mu_{\circ}, \epsilon_{\circ}$, $p_{\circ}$, and $P_{\circ}$, respectively, and $\phi$ is the negative self-gravity potential perturbation associated with the thermal ISM disk. The azimuthal momentum equation for the thermal ISM is

$$
\frac{\partial v_{\theta}}{\partial t}+\frac{V_{\theta}}{r} \frac{\partial v_{\theta}}{\partial \theta}+\frac{1}{r} \frac{d\left(r V_{\theta}\right)}{d r} v_{r}=-\frac{1}{\mu_{\circ} r} \frac{\partial p}{\partial \theta}+\frac{1}{r} \frac{\partial \phi}{\partial \theta},
$$


and in parallel, the perturbed azimuthal momentum equation for the CRG is

$$
\frac{\partial u_{\theta}}{\partial t}+\frac{V_{\theta}}{r} \frac{\partial u_{\theta}}{\partial \theta}+\frac{1}{r} \frac{d\left(r V_{\theta}\right)}{d r} u_{r}=-\frac{1}{\epsilon_{\circ} r} \frac{\partial P}{\partial \theta}+\frac{1}{r} \frac{\partial \phi}{\partial \theta} .
$$

The mass conservation for the surface mass density perturbation $\mu$ of the thermal ISM is

$$
\frac{\partial \mu}{\partial t}+\frac{1}{r} \frac{\partial\left(\mu_{\circ} r v_{r}\right)}{\partial r}+\frac{\mu_{\circ}}{r} \frac{\partial v_{\theta}}{\partial \theta}+\frac{V_{\theta}}{r} \frac{\partial \mu}{\partial \theta}=0 .
$$

The mass conservation for the mass density perturbation $\Sigma$ of the CRG is

$$
\frac{\partial \Sigma}{\partial t}+\frac{1}{r} \frac{\partial\left(\Sigma_{\circ} r u_{r}\right)}{\partial r}+\frac{\Sigma_{\circ}}{r} \frac{\partial u_{\theta}}{\partial \theta}+\frac{V_{\theta}}{r} \frac{\partial \Sigma}{\partial \theta}=0 .
$$

The three-dimensional Poisson equation for the perturbed negative self-gravity potential $\phi$ induced by $\mu$ becomes

$$
\frac{1}{r} \frac{\partial}{\partial r}\left(r \frac{\partial \phi}{\partial r}\right)+\frac{1}{r^{2}} \frac{\partial^{2} \phi}{\partial \theta^{2}}+\frac{\partial^{2} \phi}{\partial z^{2}}=-4 \pi G \mu(r, \theta) \delta(z),
$$

where the tiny contribution from the CRG to the negative self-gravity potential perturbation has been dropped, $\delta(z)$ is the Dirac delta function with $\operatorname{argument} z$, and $\mu_{\circ}$ is related to the mass density of the thermal ISM $\rho_{\circ}$ by $\rho_{\circ}(r, z) \equiv \mu_{\circ}(r) \delta(z)$. For the magnetic field perturbation $\vec{b}$, one of equations (2.3) and (2.4) may be spared when equation (2.5) is used instead. The perturbed polytropic relation for the two-dimensional ISM pressure perturbation $p$ and the surface mass density perturbation $\mu$ of the ISM becomes

$$
p=C_{S}^{2} \mu,
$$

and similarly, the perturbed two-dimensional polytropic relation for the CRG is

$$
P=C_{C}^{2} \Sigma
$$

where $C_{S}$ and $C_{C}$ are the polytropic sound speeds in the thermal ISM and in the CRG, respectively. In short, coplanar MHD density wave perturbations in the ISM disk and the bulk of the CRG are coupled electromagnetically via the large-scale mean magnetic field. ${ }^{4}$

With the $\exp (i \omega t-i m \theta)$ dependence implied for all MHD perturbation variables in equations $(2.3)-(2.13)$, where $\omega$ is the angular frequency in an inertial frame of reference and positive integer $m$ indicates the number of spiral arms, one can reduce equations $(2.3)-(2.13)$ to

$$
\begin{gathered}
r(\omega-m \Omega) b_{r}=-m B_{\theta} v_{r}, \\
i \omega b_{\theta}=\frac{\partial\left(V_{\theta} b_{r}\right)}{\partial r}-\frac{\partial\left(B_{\theta} v_{r}\right)}{\partial r},
\end{gathered}
$$

\footnotetext{
${ }^{4}$ Note that the large-scale dynamic coupling between the fluid stellar disk and the magnetized ISM gas disk is primarily gravitational (Jog \& Solomon 1984a, b; Lou \& Fan 1997, 1998b; Lou et al. 2001a).
} 


$$
\begin{aligned}
& b_{\theta}=-\frac{i}{m} \frac{\partial\left(r b_{r}\right)}{\partial r}, \\
& i(\omega-m \Omega) v_{r}-\frac{2 \Omega \mu_{\circ} v_{\theta}}{\left(\mu_{\circ}+\epsilon_{\circ}\right)}-\frac{2 \Omega \epsilon_{\circ} u_{\theta}}{\left(\mu_{\circ}+\epsilon_{\circ}\right)}+\int \frac{d z B_{\theta}}{4 \pi r\left(\mu_{\circ}+\epsilon_{\circ}\right)}\left[\frac{\partial\left(r b_{\theta}\right)}{\partial r}+i m b_{r}\right] \\
& =\frac{\partial \phi}{\partial r}-\frac{1}{\left(\mu_{\circ}+\epsilon_{\circ}\right)} \frac{\partial(p+P)}{\partial r}+\frac{(\mu+\epsilon)}{\left(\mu_{\circ}+\epsilon_{\circ}\right)^{2}} \frac{d\left(p_{\circ}+P_{\circ}\right)}{d r}, \\
& i(\omega-m \Omega) v_{\theta}+\frac{1}{r} \frac{d\left(r V_{\theta}\right)}{d r} v_{r}=-\frac{i m}{r}\left(\phi-\frac{p}{\mu_{\circ}}\right), \\
& i(\omega-m \Omega) u_{\theta}+\frac{1}{r} \frac{d\left(r V_{\theta}\right)}{d r} u_{r}=-\frac{i m}{r}\left(\phi-\frac{P}{\epsilon_{\circ}}\right), \\
& i(\omega-m \Omega) \mu+\frac{1}{r} \frac{\partial\left(r \mu_{\circ} v_{r}\right)}{\partial r}-\frac{i m \mu_{\circ}}{r} v_{\theta}=0, \\
& i(\omega-m \Omega) \Sigma+\frac{1}{r} \frac{\partial\left(r \Sigma_{\circ} u_{r}\right)}{\partial r}-\frac{i m \Sigma_{\circ}}{r} u_{\theta}=0 .
\end{aligned}
$$

The three-dimensional Poisson equation (2.11) leads to an integral representation of $\phi$ in terms of $\mu$ (Shu 1970a; Lin \& Lau 1979; Shu et al. 2000; Galli et al. 2001; Lou \& Fan 2001), which can be solved exactly for potential-density pairs in special cases (e.g., logarithmic spirals; Kalnajs 1971). In the present context, we assume a sufficiently short radial wavelength and thus invoke the tight-winding or WKBJ approximation. In this regime, it is possible to establish a local differential relation between $\phi$ and $\mu$ for the magnetized thermal gas disk of ISM (Lin \& Shu 1964, 1966; Shu 1970b). For spiral galaxies such as M51 and NGC 6946 of interest here, the tight-winding approximation is justifiable or, at least, should provide physically sensible results. We shall adopt this tight-winding approximation in our analytical analysis, as the solutions thus derived contain the information of MHD density waves in the presence of CRG fluid and of suprathermal MHD waves (see Parker 1965).

\section{DISPERSION RELATIONS OF SPIRAL MHD DENSITY WAVES}

Parker first investigated suprathermal hydromagnetic waves using the MHD perturbation equations in a thermal gas and some very hot suprathermal gas, such as interstellar cosmic rays, in a uniform magnetic field. The results of his pioneer analysis provide useful insights on our problem at hand, although the formulation here involves additional effects of disk differential rotation, self-gravity, and

a curved magnetic field in the cylindrical geometry. Parker showed that, besides an incompressible transverse Alfvén wave mode (see eq. [9] of Parker 1965), the conventional slow and fast hydromagnetic waves in the thermal gas are largely unaffected, except that there is a "hole" in the phase diagram for the fast mode in the direction perpendicular to the magnetic field (see Fig. 1 of Parker 1965; also see Appendix B here). The presence of this "hole" in the fast mode is related to the fact that bulk motions of thermal and suprathermal gases are tied together electromagnetically in the direction transverse to the magnetic field. More importantly, there is an additional suprathermal mode, representing waves in the 
suprathermal gas traveling effectively with the speed of sound in the superthermal gas alone (Appendix B).

For spiral MHD density waves in a thin self-gravitating composite gas disk in rotation, we consider compressible MHD perturbations because mass density fluctuations are coupled to gravitational potential perturbation by Poisson's equation (2.11), so that a spiral gravitational potential field exerts a dynamical influence on compressible MHD perturbations. With a cylindrical geometry and in the presence of a thin differentially rotating disk, the analysis of MHD density waves becomes more tedious (Fan \& Lou 1996; Lou \& Fan 1998; Lou et al. 2001a), yet the analogs of fast and slow MHD waves involving a suprathermal gas as described by Parker (1965) should exist on the ground of physics. We expect that for fast MHD density waves, thermal gas density and magnetic field perturbations remain more or less in phase in the tight-winding approximation, while thermal gas density and magnetic field perturbation enhancements remain significantly phase shifted for slow MHD density waves (a phase difference $\gtrsim \pi / 2$ but not exactly $\pi$ owing to the disk rotation). The existence of a somewhat modified suprathermal mode is thus anticipated as well.

To derive the dispersion relations from equations $(2.11)-(2.21)$ for the fast and slow spiral MHD density waves, we further take

$$
\phi=\Phi(r) \exp \left[i \int^{r} k(s) d s\right]
$$

where $k(r)$ is the radial wavenumber and $\Phi(r)$ is the slowly varying amplitude of the negative gravitational potential perturbation $\phi$ (Goldreich \& Tremaine 1978, 1979). One then has

$$
\frac{d \phi}{d r}=i k(r) \phi+\frac{\Phi^{\prime}(r)}{\Phi(r)} \phi
$$

where the prime over $\Phi(r)$ denotes a radial derivative. In the tight-winding regime of a large $k$, Poisson's equation (2.11) may be approximated by

$$
\left.\frac{1}{r^{1 / 2}} \frac{\partial\left(r^{1 / 2} \phi\right)}{\partial r}\right|_{z=0} \cong 2 \pi G i \operatorname{sgn}(k) \mu+\mathcal{O}\left[\frac{\mu}{(k r)^{2}}\right]
$$

which contains a fractional error on the order of $\mathcal{O}(k r)^{-2}$ (Shu 1970b; Goldreich \& Tremaine 1979), where $\operatorname{sgn}(k)=+1$ for $k>0$ and $\operatorname{sgn}(k)=-1$ for $k<0$. The cases of $k>0$ and $k<0$ correspond to leading and trailing spiral arms, respectively, and the integer $m>0$ gives the number of spiral arms. Equations (3.2) and (3.3) together give

$$
\phi=\frac{4 \pi r i G \operatorname{sgn}(k) \mu}{2 i k r+2 r \Phi^{\prime} / \Phi+1} .
$$

A substitution of equation (3.4) back into equation (3.2) gives

$$
\frac{d \phi}{d r}=\left[2 i k r+\frac{2 r \Phi^{\prime}}{\Phi}\right] \frac{2 \pi i G \operatorname{sgn}(k) \mu}{2 i k r+2 r \Phi^{\prime} / \Phi+1} \equiv \mathcal{F}_{1} \mu
$$


which defines the complex coefficient $\mathcal{F}_{1}$. From equations $(2.12),(2.18),(2.21)$ and (3.4), we can derive an equation of $\mu$ in terms of $v_{r}$ and $d v_{r} / d r$

$$
\begin{gathered}
\mu=\frac{i m \kappa^{2} \mu_{\circ} v_{r} /(2 \Omega r)+i(\omega-m \Omega) r^{-1} d\left(r \mu_{\circ} v_{r}\right) / d r}{(\omega-m \Omega)^{2}-} m^{2} C_{S}^{2} / r^{2}+\left(m^{2} / r^{2}\right) 4 \pi r i G \operatorname{sgn}(k) \mu_{\circ} /\left(2 i k r+2 r \Phi^{\prime} / \Phi+1\right) \\
\equiv \mathcal{A} v_{r}+\mathcal{B} \frac{d v_{r}}{d r}
\end{gathered}
$$

which defines the two complex coefficients $\mathcal{A}$ and $\mathcal{B}$. From equations (2.13) and (2.19) for perturbations in the CRG, one derives

$$
u_{\theta}=\frac{i u_{r}}{r(\omega-m \Omega)} \frac{d\left(r V_{\theta}\right)}{d r}+\frac{m C_{C}^{2} \Sigma}{r(\omega-m \Omega) \epsilon_{\circ}} .
$$

From mass conservation (2.21) for perturbations of the CRG, one obtains

$$
\Sigma=\frac{i m \Sigma_{\circ} \kappa^{2} u_{r} /(2 \Omega r)+i(\omega-m \Omega) r^{-1} d\left(r \Sigma_{\circ} u_{r}\right) / d r}{(\omega-m \Omega)^{2}-m^{2} C_{C}^{2} \Sigma_{\circ} /\left(r^{2} \epsilon_{\circ}\right)},
$$

where $\kappa^{2}=(2 \Omega / r) d\left(r V_{\theta}\right) / d r$ defines the epicyclic frequency $\kappa$ of the disk. From the mass conservation (2.20) for the thermal ISM, one has

$$
v_{\theta}=\frac{r(\omega-m \Omega) \mu}{m \mu_{\circ}}-\frac{i}{m \mu_{\circ}} \frac{d\left(r \mu_{\circ} v_{r}\right)}{d r} .
$$

The radial induction equation (2.14) may be arranged into the form of

$$
b_{r}=-\frac{m B_{\theta} v_{r}}{r(\omega-m \Omega)},
$$

while the divergence-free condition (2.16) of $\vec{b}$ may be written as

$$
b_{\theta}=i \frac{d}{d r}\left[\frac{B_{\theta} v_{r}}{(\omega-m \Omega)}\right]
$$

by using equation (3.10). A straightforward substitution of these equations into the radial momentum equation (2.17) leads to the following lengthy second-order ordinary differential equation (ODE)

$$
\begin{aligned}
& i(\omega-m \Omega) v_{r}-\frac{2 \Omega \mu_{\circ}}{\left(\mu_{\circ}+\epsilon_{\circ}\right)}\left[\frac{r(\omega-m \Omega)}{m \mu_{\circ}}\left(\mathcal{A} v_{r}+\mathcal{B} \frac{d v_{r}}{d r}\right)-\frac{i}{m \mu_{\circ}} \frac{d\left(r \mu_{\circ} v_{r}\right)}{d r}\right] \\
& -\frac{2 \Omega \epsilon_{\circ}}{\left(\mu_{\circ}+\epsilon_{\circ}\right)}\left\{\frac{m C_{C}^{2} \Sigma_{\circ}}{r(\omega-m \Omega) \epsilon_{\circ}}\left[\frac{i m \kappa^{2} u_{r} /(2 \Omega r)+i(\omega-m \Omega) d\left(r \Sigma_{\circ} u_{r}\right) / d r /\left(r \Sigma_{\circ}\right)}{(\omega-m \Omega)^{2}-m^{2} C_{C}^{2} \Sigma_{\circ} /\left(r^{2} \epsilon_{\circ}\right)}\right]\right. \\
& \left.+\frac{i \kappa^{2} u_{r}}{2 \Omega(\omega-m \Omega)}\right\}-\frac{i m^{2} C_{A}^{2} v_{r}}{r^{2}(\omega-m \Omega)}+i C_{A}^{2} \frac{d}{d r}\left\{r \frac{d}{d r}\left[\frac{v_{r}}{r(\omega-m \Omega)}\right]\right\} \\
& =\mathcal{F}_{1}\left(\mathcal{A} v_{r}+\mathcal{B} \frac{d v_{r}}{d r}\right)-\frac{1}{\left(\mu_{\circ}+\epsilon_{\circ}\right)} \frac{d}{d r}\left[C_{S}^{2}\left(\mathcal{A} v_{r}+\mathcal{B} \frac{d v_{r}}{d r}\right)\right] \\
& -\frac{1}{\left(\mu_{\circ}+\epsilon_{\circ}\right)} \frac{d}{d r}\left\{\frac{C_{C}^{2} \Sigma_{\circ}\left[i m \kappa^{2} u_{r} /(2 \Omega r)+i(\omega-m \Omega) d\left(r \Sigma_{\circ} u_{r}\right) / d r /\left(r \Sigma_{\circ}\right)\right]}{(\omega-m \Omega)^{2}-m^{2} C_{C}^{2} \Sigma_{\circ} /\left(r^{2} \epsilon_{\circ}\right)}\right\} \\
& +\left(\mathcal{A} v_{r}+\mathcal{B} \frac{d v_{r}}{d r}\right) \frac{1}{\left(\mu_{\circ}+\epsilon_{\circ}\right)^{2}} \frac{d\left(p_{\circ}+P_{\circ}\right)}{d r} \\
& +\frac{\Gamma C_{C}^{2} \Sigma_{\circ} /\left[(\Gamma-1) c^{2}\right]}{\left(\mu_{\circ}+\epsilon_{\circ}\right)^{2}} \frac{d\left(p_{\circ}+P_{\circ}\right)}{d r} \frac{\left[i m \kappa^{2} u_{r} /(2 \Omega r)+i(\omega-m \Omega) d\left(r \Sigma_{\circ} u_{r}\right) / d r /\left(r \Sigma_{\circ}\right)\right]}{(\omega-m \Omega)^{2}-m^{2} C_{C}^{2} \Sigma_{\circ} /\left(r^{2} \epsilon_{\circ}\right)}
\end{aligned}
$$


where $C_{A}^{2} \equiv \int d z B_{\theta}^{2} /\left[4 \pi\left(\mu_{\circ}+\epsilon_{\circ}\right)\right]$ defines the Alfvén speed $C_{A}$ in the composite disk, and the complex coefficients $\mathcal{F}_{1}, \mathcal{A}$, and $\mathcal{B}$ have already been defined earlier by equations (3.5) and (3.6) (see Appendix A for details). With $v_{r}=u_{r}$, equation (3.12) contains the information of fast and slow spiral MHD density waves as well as the suprathermal MHD mode.

\subsection{SLOW SPIRAL MHD DENSITY WAVES}

We have previously (Fan \& Lou 1996; Lou \& Fan 1998a) derived, in the tightwinding approximation, the dispersion relation for slow MHD density waves under the conditions that $\Omega \equiv V_{\theta} / r \sim$ constant, $\omega-m \Omega \sim m C_{A} / r, C_{S} \sim C_{A}, \Omega / C_{A} \sim k$, and $k r \gg 1$. Here, we require in addition that $C_{C} \gg C_{S}$ and $\Sigma_{\circ} / \mu_{\circ} \ll 1$. On the basis of Parker's analysis (Parker 1965), we expect that the basic features of slow MHD density waves should remain more or less intact and the associated CRG density fluctuation should be fairly weak. We set out to confirm these expectations by an analysis of equation (3.12) (see Appendix A) using the assumptions stated above.

To the leading order of the tight-winding approximation with $k r \gg 1$ for slow MHD density waves, the three complex coefficients $\mathcal{C}_{1}, \mathcal{C}_{2}$ and $\mathcal{C}_{3}$ in equation $(A 1)$ are approximately given by

$$
\begin{aligned}
\mathcal{C}_{1}=\frac{i C_{A}^{2}}{(\omega-m \Omega)} & +\frac{C_{S}^{2} \mu_{\circ}}{\left(\mu_{\circ}+\epsilon_{\circ}\right)} \frac{i(\omega-m \Omega)}{(\omega-m \Omega)^{2}-m^{2} \Delta / r^{2}} \\
& +\frac{i(\omega-m \Omega) C_{C}^{2} \Sigma_{\circ}}{\left(\mu_{\circ}+\epsilon_{\circ}\right)\left[(\omega-m \Omega)^{2}-m^{2} C_{C}^{2} \Sigma_{\circ} /\left(r^{2} \epsilon_{\circ}\right)\right]}, \\
\mathcal{C}_{2}= & -\frac{2 \Omega \mu_{\circ}}{\left(\mu_{\circ}+\epsilon_{\circ}\right)}\left[\frac{i r(\omega-m \Omega)^{2}}{m\left[(\omega-m \Omega)^{2}-m^{2} \Delta / r^{2}\right]}-\frac{i r}{m}\right] \\
& -\frac{2 i m \Omega C_{C}^{2} \Sigma_{\circ}}{r\left(\mu_{\circ}+\epsilon_{\circ}\right)\left[(\omega-m \Omega)^{2}-m^{2} C_{C}^{2} \Sigma_{\circ} /\left(r^{2} \epsilon_{\circ}\right)\right]} \\
& -i k \frac{2 \pi G \mu_{\circ}}{|k|} \frac{i(\omega-m \Omega)}{(\omega-m \Omega)^{2}-m^{2} \Delta / r^{2}} \\
& +\frac{C_{C}^{2} \Sigma_{\circ} i m \kappa^{2} /(2 \Omega r)}{\left(\mu_{\circ}+\epsilon_{\circ}\right)\left[(\omega-m \Omega)^{2}-m^{2} C_{C}^{2} \Sigma_{\circ} /\left(r^{2} \epsilon_{\circ}\right)\right]} \\
& +\frac{C_{S}^{2} \mu_{\circ}}{\left(\mu_{\circ}+\epsilon_{\circ}\right)} \frac{i m \kappa^{2} /(2 \Omega r)}{(\omega-m \Omega)^{2}-m^{2} \Delta / r^{2}},
\end{aligned}
$$

and

$$
\begin{aligned}
\mathcal{C}_{3}=- & \frac{\mu_{\circ}}{\left(\mu_{\circ}+\epsilon_{\circ}\right)} \frac{i(\omega-m \Omega) \kappa^{2}}{(\omega-m \Omega)^{2}-m^{2} \Delta / r^{2}}-\frac{2 \Omega \epsilon_{\circ}}{\left(\mu_{\circ}+\epsilon_{\circ}\right)}\left[\frac{i \kappa^{2}}{2 \Omega(\omega-m \Omega)}\right. \\
& \left.+\frac{m C_{C}^{2} \Sigma_{\circ}}{r(\omega-m \Omega) \epsilon_{\circ}} \frac{i m \kappa^{2} /(2 \Omega r)}{(\omega-m \Omega)^{2}-m^{2} C_{C}^{2} \Sigma_{\circ} /\left(r^{2} \epsilon_{\circ}\right)}\right] \\
& -i k \frac{2 \pi G \mu_{\circ}}{|k|} \frac{i m \kappa^{2} /(2 \Omega r)}{(\omega-m \Omega)^{2}-m^{2} \Delta / r^{2}}
\end{aligned}
$$


separately with $\Delta \equiv C_{S}^{2}-2 \pi G \mu_{\circ} /|k|$ (see Appendix A). Ignoring higher-order terms, the dispersion relation of slow MHD density waves comes out to be

$$
\begin{aligned}
(\omega-m \Omega)^{2} \cong \frac{k^{2} C_{A}^{2} m^{2} \Delta}{r^{2}}\left\{k ^ { 2 } \left[C_{A}^{2}\right.\right. & \left.+\frac{C_{S}^{2} \mu_{\circ}}{\left(\mu_{\circ}+\epsilon_{\circ}\right)}+\frac{\epsilon_{\circ} \Delta}{\left(\mu_{\circ}+\epsilon_{\circ}\right)}-\frac{2 \pi G \mu_{\circ}}{|k|}\right] \\
& \left.+\kappa^{2}\left[\frac{\mu_{\circ}}{\left(\mu_{\circ}+\epsilon_{\circ}\right)}+\frac{\epsilon_{\circ}^{2} \Delta}{C_{C}^{2} \Sigma_{\circ}\left(\mu_{\circ}+\epsilon_{\circ}\right)}\right]\right\}^{-1} .
\end{aligned}
$$

Because $\mu_{\circ} \gg \epsilon_{\circ}$ or $\Sigma_{\circ}$, the slow MHD density wave is indeed not very much affected by the presence of a tenuous CRG (Fan \& Lou 1996; Lou \& Fan 1998a, 2001). As expected, perturbation enhancements of surface mass density $\mu$ of the thermal ISM gas and parallel magnetic field $b_{\theta}$ are significantly phase shifted with a phase difference $\gtrsim \pi / 2$. The particular aspect of interest here is the phase relation and magnitude of CRG density fluctuation $\Sigma$ relative to thermal gas density fluctuation $\mu$. By equations (3.6) and (3.8), we have for large $k r$

$$
\frac{\Sigma / \Sigma_{\circ}}{\mu / \mu_{\circ}} \cong \frac{(\omega-m \Omega)^{2}-m^{2} \Delta / r^{2}}{(\omega-m \Omega)^{2}-m^{2} C_{C}^{2} \Sigma_{\circ} /\left(r^{2} \epsilon_{\circ}\right)} .
$$

For neutral or stable slow MHD density waves with $\Delta>0$, the inequality $(\omega-$ $m \Omega)^{2}<m^{2} \Delta / r^{2} \ll m^{2} C_{C}^{2} \Sigma_{\circ} /\left(r^{2} \epsilon_{\circ}\right)$ holds such that $\Sigma / \Sigma_{\circ}$ and $\mu / \mu_{\circ}$ are in phase with

$$
\frac{\Sigma}{\Sigma_{\circ}} \sim \frac{\Delta \epsilon_{\circ}}{C_{C}^{2} \Sigma_{\circ}} \frac{\mu}{\mu_{\circ}} .
$$

As $\epsilon_{\circ}$ and $\Sigma_{\circ}$ are on the same order of magnitude and $\Delta \ll C_{C}^{2}$, the relative fluctuation $\Sigma / \Sigma_{\text {。 }}$ of CRG density is much less than the relative fluctuation $\mu / \mu_{\circ}$ of thermal gas density. Therefore, the main contribution to the large-scale variation of synchrotron radio-continuum emission comes from the enhancement of magnetic field. In other words, the significant phase difference $(\gtrsim \pi / 2)$ between the enhancements of CRG density and parallel magnetic field would not reduce the contrast of synchrotron radio-continuum emission associated with slow MHD density waves in a significant manner.

As the presence of tenuous CRG does not influence the propagation of slow MHD density waves in a significant manner, it follows that the dispersion relation for slow MHD density waves in a disk with a strong differential rotation should remain more or less the same as derived earlier (Lou \& Fan 1998a).

\subsection{FAST SPIRAL MHD DENSITY WAVES}

To derive the dispersion relation of fast MHD density waves in the presence of a $\mathrm{CRG}$, we take that $(\omega-m \Omega) \sim\left(C_{S}^{2}+C_{A}^{2}\right)^{1 / 2} k, C_{S} \sim C_{A}, \Omega / C_{A} \sim k$, $k r \gg 1, C_{C} \gg C_{S}, \Sigma_{\circ} \ll \mu_{\circ}$, and $m /(k r) \gg C_{S} / C_{C}$, where the last inequality guarantees that the direction of wave propagation be sufficiently away from the radial direction. It is then straightforward to show (see Appendix A) that

$$
\begin{aligned}
\mathcal{C}_{1}=\frac{i C_{A}^{2}}{(\omega-m \Omega)} & +\frac{C_{S}^{2} \mu_{\circ}}{\left(\mu_{\circ}+\epsilon_{\circ}\right)} \frac{i(\omega-m \Omega)}{(\omega-m \Omega)^{2}-m^{2} \Delta / r^{2}} \\
& +\frac{i(\omega-m \Omega) C_{C}^{2} \Sigma_{\circ}}{\left(\mu_{\circ}+\epsilon_{\circ}\right)\left[(\omega-m \Omega)^{2}-m^{2} C_{C}^{2} \Sigma_{\circ} /\left(r^{2} \epsilon_{\circ}\right)\right]},
\end{aligned}
$$


where the third term on the right-hand side due to the CRG is small,

$$
\mathcal{C}_{2}=k \frac{2 \pi G \mu_{\circ}}{|k|} \frac{(\omega-m \Omega)}{(\omega-m \Omega)^{2}-m^{2} \Delta / r^{2}},
$$

and

$$
\begin{aligned}
\mathcal{C}_{3}=i(\omega & -m \Omega)-\frac{i \mu_{\circ}}{\left(\mu_{\circ}+\epsilon_{\circ}\right)} \frac{(\omega-m \Omega) \kappa^{2}}{(\omega-m \Omega)^{2}-m^{2} \Delta / r^{2}}-\frac{\epsilon_{\circ} i \kappa^{2}}{\left(\mu_{\circ}+\epsilon_{\circ}\right)(\omega-m \Omega)} \\
& -\frac{2 \Omega}{\left(\mu_{\circ}+\epsilon_{\circ}\right)} \frac{m C_{C}^{2} \Sigma_{\circ}}{r(\omega-m \Omega)} \frac{i m \kappa^{2} /(2 \Omega r)+i(\omega-m \Omega) d \ln \left(r \Sigma_{\circ}\right) / d r}{(\omega-m \Omega)^{2}-m^{2} C_{C}^{2} \Sigma_{\circ} /\left(r^{2} \epsilon_{\circ}\right)}
\end{aligned}
$$

in equation $(A 1)$. The dispersion relation of fast spiral MHD density waves is thus given by

$$
(\omega-m \Omega)^{2} \cong \frac{\mu_{\circ} \kappa^{2}}{\left(\mu_{\circ}+\epsilon_{\circ}\right)}+k^{2}\left[C_{A}^{2}+\frac{C_{S}^{2} \mu_{\circ}}{\left(\mu_{\circ}+\epsilon_{\circ}\right)}-\frac{2 \pi G \mu_{\circ}}{|k|}\right] .
$$

As expected, the presence of CRG does not affect very much the propagation of fast MHD density waves as long as $m /(k r) \gg C_{S} / C_{C}$. This inequality guarantees that a propagation of fast MHD density waves is sufficiently away from the radial

direction that is perpendicular to the background magnetic field (see Appendix B). One can readily show that the perturbation enhancements of thermal gas density and parallel magnetic field are largely in phase to the leading order of large $k r$. From equation (3.17), it is clear that the perturbation enhancements of thermal ISM density and CRG density are out of phase (i.e., a phase difference of $\sim \pi$ ). Furthermore,

$$
\frac{\Sigma / \Sigma_{\circ}}{\mu / \mu_{\circ}} \sim-\frac{k^{2} r^{2} C_{A}^{2}}{m^{2} C_{C}^{2}},
$$

which gives a much smaller $\Sigma / \Sigma_{\circ}$ relative to $\mu / \mu_{\circ}$. Nevertheless, in comparison to the case of slow MHD density waves, $\Sigma / \Sigma_{\circ}$ is larger by a factor $k^{2} r^{2} / m^{2}$ in the case of fast MHD density waves. Although perturbation enhancements of CRG density and parallel magnetic field are out of phase, the synchrotron radiocontinuum emission is dominantly determined by the perturbation enhancement of large-scale parallel magnetic field.

\subsection{THE SUPRATHERMAL MODE}

To derive the dispersion relation of the suprathermal mode, we take that $(\omega-m \Omega) \sim m C_{C} / r, C_{S} \sim C_{A}, \Omega / C_{A} \sim k, C_{C} \gg C_{S}, \Sigma_{\circ} \ll \mu_{\circ}$, and $k r \gg 1$. It follows that in equation $(A 1)$

$$
\begin{aligned}
\mathcal{C}_{1}=\frac{i C_{A}^{2}}{(\omega-m \Omega)} & +\frac{C_{S}^{2} \mu_{\circ}}{\left(\mu_{\circ}+\epsilon_{\circ}\right)} \frac{i(\omega-m \Omega)}{(\omega-m \Omega)^{2}-m^{2} \Delta / r^{2}} \\
& +\frac{i(\omega-m \Omega) C_{C}^{2} \Sigma_{\circ}}{\left(\mu_{\circ}+\epsilon_{\circ}\right)\left[(\omega-m \Omega)^{2}-m^{2} C_{C}^{2} \Sigma_{\circ} /\left(r^{2} \epsilon_{\circ}\right)\right]}
\end{aligned}
$$




$$
\mathcal{C}_{2}=k \frac{2 \pi G \mu_{\circ}}{|k|} \frac{(\omega-m \Omega)}{(\omega-m \Omega)^{2}-m^{2} \Delta / r^{2}},
$$

and

$$
\mathcal{C}_{3}=i(\omega-m \Omega)-\frac{i \mu_{\circ}}{\left(\mu_{\circ}+\epsilon_{\circ}\right)} \frac{(\omega-m \Omega) \kappa^{2}}{(\omega-m \Omega)^{2}-m^{2} \Delta / r^{2}}
$$

(see Appendix A). By dropping several higher-order terms, we arrive at

$$
\begin{aligned}
(\omega & -m \Omega)^{4}-\left\{\frac{\mu_{\circ} \kappa^{2}}{\left(\mu_{\circ}+\epsilon_{\circ}\right)}+k^{2}\left[C_{A}^{2}+\frac{C_{S}^{2} \mu_{\circ}}{\left(\mu_{\circ}+\epsilon_{\circ}\right)}-\frac{2 \pi G \mu_{\circ}}{|k|}\right]\right. \\
& \left.+\frac{k^{2} C_{C}^{2} \Sigma_{\circ}}{\left(\mu_{\circ}+\epsilon_{\circ}\right)}+\frac{m^{2} C_{C}^{2} \Sigma_{\circ}}{r^{2} \epsilon_{\circ}}\right\}(\omega-m \Omega)^{2} \\
& +\left\{\frac{\mu_{\circ} \kappa^{2}}{\left(\mu_{\circ}+\epsilon_{\circ}\right)}+k^{2}\left[C_{A}^{2}+\frac{C_{S}^{2} \mu_{\circ}}{\left(\mu_{\circ}+\epsilon_{\circ}\right)}-\frac{2 \pi G \mu_{\circ}}{|k|}\right]\right\} \frac{m^{2} C_{C}^{2} \Sigma_{\circ}}{r^{2} \epsilon_{\circ}} \cong 0,
\end{aligned}
$$

which contains both the suprathermal wave mode and the fast MHD density wave mode. If the term $(\omega-m \Omega)^{2} k^{2} C_{C}^{2} \Sigma_{\circ} /\left(\mu_{\circ}+\epsilon_{\circ}\right)$ were absent, then relation (3.27) may be readily expressed as a multiplication of two simple factors. To the present level of approximation, the dispersion relation for the suprathermal mode is approximately given by

$$
(\omega-m \Omega)^{2} \cong \frac{m^{2} C_{C}^{2} \Sigma_{\circ}}{r^{2} \epsilon_{\circ}}+\frac{k^{2} C_{C}^{2} \Sigma_{\circ}}{\left(\mu_{\circ}+\epsilon_{\circ}\right)}+\frac{\mu_{\circ} \kappa^{2}}{\left(\mu_{\circ}+\epsilon_{\circ}\right)}+k^{2}\left[C_{A}^{2}+\frac{C_{S}^{2} \mu_{\circ}}{\left(\mu_{\circ}+\epsilon_{\circ}\right)}-\frac{2 \pi G \mu_{\circ}}{|k|}\right] .
$$

Again, from the relation (3.17), it is seen that $\Sigma / \Sigma_{\circ}$ and $\mu / \mu_{\circ}$ are in phase with

$$
\frac{\Sigma}{\Sigma_{\circ}} \sim \frac{C_{C}^{2} m^{2}}{C_{S}^{2} k^{2} r^{2}} \frac{\mu}{\mu_{\circ}}
$$

where the magnitude of $\Sigma / \Sigma_{\circ}$ may be comparable to or larger than that of $\mu / \mu_{\circ}$ for the suprathermal mode.

The velocity perturbations of the suprathermal mode can be derived from the following relationship

$$
\begin{aligned}
u_{\theta}=\frac{m C_{C}^{2} \Sigma_{\circ}}{r(\omega-m \Omega) \epsilon_{\circ}} & \frac{i m \kappa^{2} u_{r} /(2 \Omega r)+i(\omega-m \Omega)\left[u_{r} d \ln \left(r \Sigma_{\circ}\right) / d r+d u_{r} / d r\right]}{(\omega-m \Omega)^{2}-m^{2} C_{C}^{2} \Sigma_{\circ} /\left(r^{2} \epsilon_{\circ}\right)} \\
& +\frac{i \kappa^{2} u_{r}}{2 \Omega(\omega-m \Omega)} .
\end{aligned}
$$

For an order-of-magnitude estimates, we have

$$
\frac{u_{\theta}}{u_{r}} \sim-\frac{m C_{C}^{2} \Sigma_{\circ}}{k r C_{S}^{2} \epsilon_{\circ}}
$$

For $u_{r} \sim 1 \mathrm{~km} \mathrm{~s}^{-1}$, the azimuthal velocity perturbation $u_{\theta}$ could be as large as $\sim 10^{4} \mathrm{~km} \mathrm{~s}^{-1}$. For $u_{\theta} \gtrsim C_{A}$, one should be seriously concerned with streaming instabilities along the magnetic field. Parker (1969) pointed out the possibility of Landau damping for the suprathermal mode in the CRG. 


\section{APPLICATIONS TO SPIRAL GALAXIES}

To apply the results of our analyses to large-scale spiral structures of gasrich disk galaxies seen in synchrotron radio-continuum emissions, one needs to have an idea for the plausible state of CRG in a typical magnetized disk galaxy. If the background CRG as a whole (either a fat disk or an oblated spheroid) largely corotates with the stellar and magnetized gas disks while retains largescale axisymmetry, then our theoretical results indicate the following. Firstly, in the presence CRG density fluctuations on large spatial scales, the suprathermal mode propagates essentially with the cosmic-ray sound speed (always less than but very close to the speed of light $c$ ) more or less along magnetic field lines to smooth out CRG fluctuations. In this case, the large-scale distribution of CRG is likely to vary in the radial direction due to the presence of a mean azimuthal galactic magnetic field. Secondly, CRG density fluctuations associated with slow MHD density waves are extremely weak as compared to thermal gas density fluctuations. While the perturbation enhancements of CRG density and parallel magnetic field are significantly phase shifted (i.e., $\gtrsim \pi / 2$ ), the enhancement in synchrotron radio-continuum emission is mainly determined by the enhancement of parallel magnetic field. Thirdly, CRG density fluctuations associated with fast MHD density waves are also sufficiently weaker than thermal gas density fluctuations to warrant that the enhancement of synchrotron radio-continuum emission basically goes with the enhancement of parallel magnetic field; the latter anti-correlates with the minute enhancement of CRG density fluctuation.

For an actual spiral galaxy, several complications and uncertainties arise. The key question ties to the actual distribution of relativistically hot CRG in a typical disk spiral galaxy. We further elaborate several relevant aspects below.

1. It is known observationally that the total luminosities of infrared and nonthermal radio-continuum emissions from spiral galaxies are tightly correlated (Dickey \& Salpeter 1984; Helou et al. 1985; Helou \& Bicay 1993). This empirical fact leads to the important notion that somehow the formation of massive stars and the production of relativistic cosmic rays are correlated through a chain of complex physical processes yet to be explored and understood. For example, an active region of massive star formation may produce, perhaps in the statistical sense, more explosions of type II supernovae that are favored sources of cosmic ray production (up to energy of $\sim 10^{15} \mathrm{eV}$ ). It is also plausible that MHD termination shocks confining fast stellar winds from numerous young $\mathrm{O}, \mathrm{B}$ stars sustain the supply of galactic cosmic rays in a significant manner. It thus seems sensible that on large spatial scales, optical spiral gas arms of relatively high densities should contain more sources for cosmic ray production.

2. Interstellar cloud complexes, where young massive stars are continuously forming on smaller scales, are generally thought to be initially triggered by Parker instability (i.e., magnetic Rayleigh-Taylor instability, see Parker 1966, 1969; Shu 1974; Mouschovias 1996) which involves downward slide of condensed thermal gas into magnetic valleys and upward expansions of magnetic flux tubes further inflated by CRG in the dimension perpendicular to the galactic disk. To account for such processes, one must allow for MHD fluctuations perpendicular to a galactic 
plane. The development of Parker instability disturbs galactic magnetic field on horizontal size scales of $\sim 1 \mathrm{kpc}$ or so. Subsequent activities of star formation can lead to intensified but disordered magnetic fields on smaller spatial scales via ISM turbulence. Therefore, the total magnetic field strength may increase with star formation activities in high-density gas arms. As enhanced magnetic fields in this manner tend to be disordered, the degree of polarization in radio-continuum emissions should be correspondingly low. Therefore for fast MHD density waves with in-phase gas density and magnetic field enhancements, manifestations of total and polarized radio-continuum emissions are in competition. That is, along a highdensity gas arm with strong star formation activities, the total radio-continuum emission would be very strong whereas the degree of polarization would become weak. For moderate star formation activities along high-density gas arms, total emissions are generally stronger than polarized emissions but one may still detect degrees of polarizations. For both M51 (Neininger 1992) and NGC 2997 (Han et al. 1999), polarized radio intensity peaks closely follow the inner edges of optical spiral arms and coincide with narrow dark dust lanes. For M31 (Beck et al. 1980), magnetic "torus" coincide with the "ring" (for the controversy of "ring" or tightwinding spiral arms for M31, the reader is referred to references in Berkhuijsen et al. 2000). On the other hand, for slow MHD density waves with phase-shifted gas density and magnetic field enhancements, polarized radio intensity arms are much less affected by star formation activities along high-density gas arms where total radio emissions still remain to be strong. In terms of overall structural patterns, NGC 6946 fits this scenario well (Lou \& Fan 2002).

3. Observations indicate that large-scale galactic magnetic field normally lies more or less in the disk plane of a spiral galaxy. Within the disk of a nearly faceon spiral galaxy (e.g., M51, NGC 2997 etc), polarized radio-continuum emissions reveal a global swirl-like pattern in the same sense of optical spiral arms (Neininger 1992; Berkhuijsen et al. 1997; Han et al. 1999). ${ }^{5}$ The usual impression that magnetic field remains aligned and connected along spiral arms would be difficult to reconcile with the existence of a strong disk differential rotation (LyndenBell 1966; Roberts \& Yuan 1970). Disk differential rotation would wrap up a connected magnetic field line along a spiral arm in a few turns of galactic rotation. Similar to the winding dilemma of material spiral arms (Goldreich \& LyndenBell 1965; Lin \& Shu 1966), this winding dilemma for the magnetic field may be resolved by considering large-scale azimuthal galactic magnetic field strongly distorted by galactic MHD density waves in a systematic manner; it is plausible that enhanced magnetic field locally orients roughly along a spiral arm but is not actually connected along the spiral arm. In this scenario, magnetic spiral arms appear as a result of the collective behavior of phase-organized, distorted rings of magnetic field and gas stream lines ${ }^{6}$. We note that M51 seen in linearly polarized optical emissions shows a more circular or circumferential pattern of magnetic field configuration (e.g., Scarrott, Ward-Thompson \& Warren-Smith 1987).

4. For the scenario of enhanced magnetic field aligned and connected along a

${ }^{5}$ At longer radio wavelengths, the effect of Faraday rotation gives rise to more distorted or irregular swirl-like patterns (e.g., Berkhuijsen et al. 1997).

6 This is very much like the kinematic description of density waves in a stellar disk by visualizing the collective behavior of phase-organized, distorted rings of mean stellar orbits as suggested by Kalnajs (1973). 
high-density spiral gas arm, cosmic rays produced by star formation and type II supernovae would be somewhat confined to retain relatively high number density along the arm. While for the scenario of distorted rings of magnetic field associated with MHD density waves, cosmic rays produced in high gas density spiral arms can redistribute themselves more readily along distorted rings of magnetic field, maintaining a large-scale radial gradient across rings of magnetic field at the same time. For a mature late-type spiral galaxy, the level of cosmic rays has probably reached the maximum that a galaxy can contain and remains more or less steady (i.e., leakage and decays are globally balanced by production of cosmic rays). In the azimuthal direction, a relatively high concentration of cosmic rays can be smoothed out via propagation of the suprathermal mode (Parker 1965, 1969), by various diffusion processes (see Cesarsky 1980) and by scattering processes.

5. It is the more common situation that the spiral pattern of the total (random and organized magnetic fields plus cosmic rays) synchrotron radio-continuum emission more or less coincides with the optical spiral pattern in nearby spiral galaxies. Typically, a spiral arm of total synchrotron radio-continuum emission is broader and may extend further outside optical arms. This demonstrates the ability of cosmic rays to diffuse across magnetic field and to infiltrate a much larger spatial volume than their original sources of production. Qualitatively, one tends to interpret the optical-radio spiral pattern correlation in terms of somewhat high concentration of cosmic rays and enhanced random magnetic fields along a spiral arm of high thermal gas density where stars are actively forming. As large-scale organized magnetic field may not be completely disrupted along star-forming regions, coherent structures in polarized radio-continuum emissions can be still detected.

6. In addition to the fact that total luminosities of infrared and synchrotron radio-continuum emission from spiral galaxies are tightly correlated, new evidence is forthcoming for the infrared-radio correlation in spiral structural patterns within disk galaxies (e.g., M31, see references in Berkhuijsen et al. 2000 and Lou \& Fan 2000a, b), which is quite similar to optical-radio correlation in spiral patterns. With increasing sensitivity and angular resolutions of infrared observations, we expect more examples of detailed spiral arm correlations. This trend of infraredradio correlation in spiral patterns would be consistent with the general perception of spiral MHD density waves, even though the chain of physical processes on various scales that lead to such correlated radiative manifestations remain to be thoroughly understood.

7. In identifying fast MHD density waves with large-scale optical and radio spiral patterns in M51 (Fan \& Lou 1996; Lou \& Fan 1998a), we follow a few clues. Firstly, both polarized intensity maps and degree-of-polarization maps for synchrotron radio-continuum emissions from M51 (Neininger 1992; Berkhuijsen et al. 1997) reveal a global spiral pattern well correlated with the optical spiral pattern. This is the strongest evidence that large-scale galactic magnetic fields are involved in the dynamics of density waves. Secondly, both polarized intensity and degree of polarization are usually strong along optical spiral arms where thermal gas concentration is relatively high. The coincident enhancement of thermal gas density and magnetic field is expected from the basic property of fast MHD density waves. This implies that the process of star formation does not completely disrupt large-scale galactic magnetic field, although one cannot tell immediately whether 
CRG density is much higher in spiral arms than in interarm regions. Even if CRG density distributes rather evenly in the azimuthal direction, it is still expected to see enhanced polarized and total radio-continuum intensities. Finally, from the perspective of self excitation and maintenance of MHD density waves ${ }^{7}$, fast MHD density waves are preferentially swing amplified in a disk of strong differential rotation (Fan \& Lou 1997) which is the case for M51 as well as for NGC 2997.

8. In identifying slow MHD density waves with large-scale optical and radio spiral patterns in the spiral galaxy NGC 6946 (Beck \& Hoernes 1996; Beck et al. 1996; Fan \& Lou 1996; Ferguson et al. 1998; Frick et al. 2000; Lou \& Fan 1998a, 2002), we would like to clarify a few points. Firstly, although somewhat fuzzy, large-scale radio spiral pattern in NGC 6946 again indicates the involvement of magnetic field in global density waves. The total synchrotron radio-continuum emission along optical spiral arms is strong (see Fig. 3 of Beck \& Hoernes 1996). This can be explained, as usual, in terms of the enhancement of small-scale random magnetic fields and cosmic ray production along zones of relatively high thermal gas concentration where stars are continuously borne. Secondly, intensity and degree of polarized radio emission are sufficiently strong to reveal global magnetic arms interlaced with optical spiral $\operatorname{arms}^{8}$ (see Fig. 2 of Beck \& Hoernes 1996 and Frick et al. 2000). For slow MHD density waves (Fan \& Lou 1996; Lou \& Fan 1998a), the enhancements of parallel magnetic field and thermal gas density are significantly phase shifted (i.e., $\gtrsim \pi / 2$ ), and the associated fluctuation of CRG density is so weak as can be neglected. It is therefore natural to interpret the prominent magnetic arms in polarized radio-continuum intensity in terms of enhancements of large-scale organized magnetic field, associated with slow MHD density waves, submerged in an omnipresent CRG. In this scenario, magnetic arms are interlaced with the optical spiral arms as a result of significant phase shift between enhancements of parallel magnetic field and thermal gas density. The fact that the polarized radio intensity is extremely low along optical arms (or total radio intensity arms; see Fig. 3 of Beck \& Hoernes 1996) may be due to a nearly complete disruption of an already weakened large-scale magnetic field or due to an almost flat distribution of $\mathrm{CRG}$ along the azimuthal direction ${ }^{9}$ or both. At least, the CRG density cannot be too much higher across optical arms than in interarm regions; otherwise, the presence of a weak organized large-scale magnetic field may lead to a polarized radio-continuum intensity peak within optical arms as well.

9. We have developed very recently (Lou \& Fan 2002; Lou 2002) models for stationary fast and slow MHD density waves with logarithmic spirals in a mag-

7 The presence of a companion (NGC 5195) around M51 (NGC 5194) is probably responsible only for the distortion in the outer spiral pattern as a result of tidal interaction (Toomre \& Toomre 1972; Elmegreen et al. 1989); the inner spiral pattern may be self-sustained (Rix \& Rieke 1993; Lin 1996).

8 Even in the interarm regions, total radio-continuum emission is several times stronger than the peak polarized radio-continuum intensity. However, an interarm bump in total radio emission can be attributed to polarized intensity peak.

9 That is, CRG density enhancement across star-forming optical arms may be smoothed out sufficiently fast such that the distribution remains more or less uniform in the azimuthal direction along magnetic field lines. 
netized singular isothermal disk (MSID) with a flat rotation curve. The Poisson equation can be solved exactly without invoking the tight-winding approximation (Kalnajs 1971; Shu et al. 2000) so that the pitch angle of a logarithmic spiral can be arbitrary. Our main motivation is to address the issue of sustaining an extended slow MHD density wave pattern within a disk of a flat rotation curve (Sofue 1996; Ferguson et al. 1998; Frick et al. 2000). Given what we have learned from this analysis, it is possible to further include a CRG into the formalism of Lou \& Fan (2002). It is expected that minute enhancements of logarithmic spiral arms in the CRG would be phase-shifted relative to enhancements of logarithmic spiral arms of magnetic field for either fast and slow MHD density waves. An additional stationary MSID configuration with logarithmic arms is also expected, corresponding to the suprathermal mode (Parker 1965).

\section{NOTES AND DISCUSSIONS}

We have studied fast and slow MHD density waves in a rotating magnetized thermal gas disk in the presence of CRG that is treated as a tenuous, relativistically hot gas fluid for large-scale and low-frequency dynamic phenomena. In this formalism, thermal gas and CRG are tied to the galactic magnetic field in transverse bulk motions but are allowed to move relative to each other along magnetic field lines. One way to better appreciate fast and slow MHD density waves (Fan \& Lou 1996; Lou \& Fan 1998a; Lou et al. 2001a; Lou 2002) is to first understand the basic properties of fast and slow MHD waves in a uniformly magnetized medium. Similarly, the way to fully appreciate fast and slow MHD density waves in the presence of CRG in the current context is to acquaint oneself with the basic properties of MHD wave modes in a uniformly magnetized thermal medium submerged in a uniform CRG pioneered by Parker (Parker 1965, 1967, 1969; see also Appendix B for more specifics).

The analysis of Parker (1965) leads to following basic theoretical facts. First, the conventional slow MHD wave in the thermal gas is not very much affected by the presence of CRG, and the associated CRG mass density fluctuation, which anti-correlates with the parallel magnetic field perturbation (Appendix B), is tiny compared to the thermal gas density fluctuation. Secondly, the conventional fast MHD wave in the thermal gas, propagating sufficiently away from the direction perpendicular to the background magnetic field, is not very much affected by the presence of CRG, and the associated CRG mass density fluctuations, which also anti-correlates with the parallel magnetic field perturbation, is also tiny compared to the thermal gas density fluctuation. In the propagation direction perpendicular to the magnetic field, the conventional fast MHD wave is suppressed (see Fig. 1 of Parker 1965). And finally, there exists a suprathermal mode resulting primarily from the compression of a relativistically hot CRG with a characteristic sound speed close to the speed of light $c$. Enhancements of CRG density, parallel magnetic field, and thermal gas density are all in phase for the suprathermal mode.

The specific aims of performing this analysis are to derive the phase relationships between the perturbation enhancements of parallel magnetic field and CRG

mass density associated with fast and slow MHD density waves, to confirm that 
the phase relationships between the perturbation enhancements of parallel magnetic field and thermal gas density associated with fast and slow MHD density waves in the presence of CRG remain more or less the same as in the case where CRG is absent (Fan \& Lou 1996; Lou \& Fan 1998a), and to assess the net effect on synchrotron radio-continuum emissions as a result of fluctuations in parallel magnetic field strength and in CRG mass density.

The basic conclusions of our analysis are the following. (1) In the presence of CRG, fast and slow MHD density waves are only slightly modified, and there exists an additional generalized suprathermal mode. (2) As expected, the perturbation enhancements of parallel magnetic field and the thermal gas are in phase for fast MHD density waves and are significantly phase shifted (i.e. $\gtrsim \pi / 2$ ) for slow MHD density waves. (3) The perturbation enhancements of the thermal gas and CRG mass density are in phase for slow MHD density waves and are signifcantly phase shifted (i.e., $\gtrsim \pi / 2$ ) for fast MHD density waves; in other words, the perturbation enhancements of parallel magnetic field and CRG mass density are significantly phase shifted (i.e., $\gtrsim \pi / 2$ ) for both fast and slow MHD density waves. (4) As CRG mass density fluctuation associated fast and slow MHD density waves is tiny, the radio-continuum structures seen in synchrotron emissions are primarily determined by magnetic field enhancements.

One restriction of our analysis is to require that velocity and magnetic field perturbations lie within the gas disk plane. This constraint, which leads to a great deal of simplification in the analysis, does exclude the possible occurrence of Parker instability (i.e., magnetic Rayleigh-Taylor instability) in the vertical direction across the magnetized disk (Parker 1966, 1967; Shu 1974; Mouschovias 1996 and extensive references therein). The Parker instability gives rise to magnetic loops or bubbles inflated by cosmic rays on both sides of the disk and causes condensation or conglomeration of thermal gas clouds into magnetic valleys on spatial scales in the order of $\sim 1 \mathrm{kpc}$ (give-and-take a factor of 2 ) along the mean magnetic field. As a result of vertical undulation of magnetic field lines and redistribution of CRG, synchrotron intensity can vary on the scale of $\sim 1 \mathrm{kpc}$. Since fast and slow MHD density waves vary on spatial scales $\gtrsim$ several kpc, the overall enhancement of synchrotron emission resulting from transverse compression of large-scale magnetic field within the disk will not be fundamentally altered by relatively small scale magnetic bubbles pertruding out of the disk plane. In short, even though quantitative calculations of synchrotron contrast must take consequences of Parker instability into account, the enhancement of large-scale galactic magnetic field plays a dominant role for increasing synchrotron radio-continuum intensity.

Another restriction of our analysis is to require that waves propagate within the disk plane, with vertical wavenumber $k_{z}=0$. As noted recently in Lou et al. (2001a), this assumption excludes a class of magnetorotational instabilities (MRIs) given the specified disk and field geometries (e.g., Balbus \& Hawley 1992; Terquem \& Papaloizou 1996; Kim \& Ostriker 2000). The well-known axisymmetric MRIs (Velikhov 1959; Chandrasekhar 1960, 1961; Balbus \& Hawley 1991, 1998) involve a disk differential rotation, a weak magnetic field component parallel to the rotation axis, and vertical perturbation variations with $k_{z} \neq 0$. For nonaxisymmetric MRIs in a disk with a toroidal magnetic field, it is essential to realize the fact $k_{z} \neq 0$ that couples the perturbation equations for velocity and magnetic field perturbations 
perpendicular to the disk and the equations for MHD perturbations coplanar with the disk (Lou et al. 2001a).

\section{ACKNOWLEDGMENTS}

We are grateful to E. N. Parker for insightful discussions. This research has been supported in part by grants from the US NSF (ATM-9320357, AST-9731623) to the University of Chicago, by the ASCI Center for Astrophysical Thermonuclear Flashes at the University of Chicago under Department of Energy contract B341495, by the Visiting Scientist Programs at the Institute of Astronomy and Astrophysics, Academia Sinica (NSC-88-2816-M-001-0010-6 and NSC89-2816-M0010006-6) and at the National Taiwan University (NSC89-2112-M002-037), by the Yangtze Endowment through the Tsinghua University, by the Special Funds for Major State Basic Science Research Projects of China, and by the Collaborative Research Fund from the NSF of China (NSFC) for Young Outstanding Overseas Chinese Scholars (NSFC 10028306) at the National Astronomical Observatory, Chinese Academy of Sciences.

\section{REFERENCES}

Bardeen J. M., 1975, in Dynamics of Stellar Systems, IAU Symposium No. 69, ed. Hayli A., p. 297, Reidel, Dordrecht.

Balbus S. A., Hawley J. F., 1991, ApJ, 376, 214

Balbus S. A., Hawley J. F., 1992, ApJ, 400, 610

Balbus S. A., Hawley J. F., 1998, Rev. Mod. Phys., 70, 1

Beck R., Hoernes P., 1996, Nature, 379, 47

Beck R., Brandenburg A., Moss D., Shukurov A., Sokoloff D., 1996, ARA\&A, 34, 155

Beck, R. et al. 1999, Nature, 397, 324

Berkhuijsen E. M., Wielebinski R., eds, 1978, Structure and Properties of Nearby Galaxies, IAU Symposium No. 77, Reidel, Dordrecht

Berkhuijsen E. M., Horellou C., Krause M., Neininger N., Poezd A. D., Shukurov A., Sokoloff D. D., 1997, A\&A, 318, 700

Berkhuijsen E. M., Beck R., Walterbos R. A. M., eds. 2000, 232 WE-Heraeus Seminar on The Interstellar Medium in M31 and M33 (Aachen: Shaker Verlag)

Bertin G., Lin C. C., Lowe S. A., Thurstans R. P., 1989a, ApJ, 338, 78

Bertin G., Lin C. C., Lowe S. A., Thurstans R. P., 1989b, ApJ, 338, 104

Bertin G., Lin, C. C., 1996, Spiral Structure in Galaxies: A Density Wave Theory. MIT Press, Cambridge, Mass. 
Bicay M. D., Helou G., 1990, ApJ, 362, 59

Binney J., Tremaine S., 1987, Galactic Dynamics. Princeton University Press, Princeton, New Jersey

Cesarsky C. J., 1980, ARA\&A, 18, 289

Chandrasekhar, S. 1960, Proc. Nat. Acad. Sci., 46, 253

Chandrasekhar S., 1961, Hydrodynamic and Hydromagnetic Stability. Dover Publications, New York

Condon J. J., Anderson M. L., Helou G., 1991, ApJ, 376, 95

Crosthwatte L. P., Turner J. L., Ho P. T. P., 2000, AJ, 119, 1720

de Jong T., Klein U., Wielebinski R., Wunderlich E., 1985, A\&A, 147, L6

Dickey J. M., Salpeter E. E., 1984, AJ, 284, 461

Elmegreen B. G., 1987, ApJ, 312, 626

Elmegreen, B. G. 1994, ApJ, 425, L73

Elmegreen B. G., 1995, MNRAS, 275, 944

Elmegreen B. G., Elmegreen D. M., Seiden P., 1989, ApJ, 343, 602

Elmegreen D. M., 1981, ApJ Suppl., 47, 229

Elmegreen D. M., Chromey F. R., Warren A. R., 1998, AJ, 116, 2834

Fan Z. H., Lou Y.-Q., 1996, Nature, 383, 800

Fan Z. H., Lou Y.-Q., 1997, MNRAS, 291, 91

Fan Z. H., Lou Y.-Q., 1999, MNRAS, 307, 645

Ferguson A. M. N., Wyse R. F. G., Gallagher J. S., Hunter D. A., 1998, ApJ, 506, L19

Frick P., Beck R., Shukurov A., Sokoloff D., Ehle M., Kamphuis J., 2000, MNRAS, 318,925

Galli D., Shu F. H., Laughlin G., Susana L., 2001, ApJ, 551, 367

Goldreich P., Lynden-Bell D., 1965, MNRAS, 130, 97

Goldreich P., Tremaine S., 1978, ApJ, 222, 850

Goldreich P., Tremaine S., 1979, ApJ, 233, 857

Greisen K., 1960, Ann. Rev. Nuclear Science, 10, 62

Han, J. L., Beck R., Ehle M., Haynes R. F., Wielebinski R., A\&A, 1999, 348, 405

Harwit M., Pacini F., 1975, ApJ, 200, L127

Helou G., 1991, in The Interpretation of Modern Synthesis Observations of Spiral Galaxies, ed. N. Duric and Crane P. C. Astronomical Society of the Pacific Conference Series, vol 18. pp. 125-133

Helou G., Soifer B. T., Rowan-Robinson M., 1985, ApJ, 298, L7

Helou G., Bicay M. D., 1993, ApJ, 415, 93 
Helou G., et al., 1996, A\&A, 315, L157

Hohl F., 1971, ApJ, 168, 343

Jog C. J., Solomon P. M., 1984a, ApJ, 276, 114

Jog C. J., Solomon P. M., 1984b, ApJ, 276, 127

Jog C. J., 1992, ApJ, 390, 378

Jog C. J., 1996, MNRAS, 278, 209

Julian W. H., Toomre A., 1966, ApJ, 146, 810

Kalnajs A. J., 1971, ApJ, 166, 275

Kalnajs A. J., 1973, Proc. Astron. Soc. Australia, 2, 174

Kennicutt R. C. Jr., 1989, ApJ, 344, 685

Kennicutt R. C., Tamblyn P., Congdon C. W., 1994, ApJ, 435, 22

Kent S. M., 1986, AJ, 91, 1301

Kent S. M., 1987, AJ, 93, 816

Kent S. M., 1988, AJ, 96, 514

Kim W.-T., Ostriker E. C., 2000, ApJ, 540, 372

Kormendy J., Norman C. A., 1979, ApJ, 233, 539

Krause M., 1993, in The Cosmic Dynamo, IAU Symposium No. 157, eds. Krause F., Rädler K. H., Rüdiger G. pp. 305-310, Kluwer Academic, Dordrecht.

Krause M., Hummel E., Beck R., 1989, A\&A, 217, 4

Lerche I. 1967, ApJ, 149, 395, 533

Lerche I., Parker E. N., 1966, ApJ, 145, 106

Lin C. C., 1967a, in Relativity Theory and Astrophysics, vol. 2, Galactic Structure. ed. Ehlers J., pp. 66-97, Am. Math. Soc., Providence, R. I.

Lin C. C., 1967b, ARA\&A, 5, 453

Lin C. C., 1987, Selected Papers of C. C. Lin. World Scientific, Singapore.

Lin C. C., Shu F. H., 1964, ApJ, 140, 646

Lin C. C., Shu F. H., 1966, Proc. Natl. Acad. Sci. U.S.A., 55, 229

Lin C. C., Lau Y. Y., 1979, Studies Appl. Math., 60, 97

Lou Y.-Q., 1996a, MNRAS, 279, L67

Lou Y.-Q., 1996b, MNRAS, 279, 129

Lou Y.-Q., 2002, MNRAS, 337, 225

Lou Y.-Q., Fan Z. H., 1997, Communications in Nonlinear Science and Numerical Simulation, 2 (No. 2), 59

Lou Y.-Q., Fan Z. H., 1998a, ApJ, 493, 102

Lou Y.-Q., Fan Z. H., 1998b, MNRAS, 297, 84 
Lou Y.-Q., Fan Z. H., 2000a, MNRAS, 315, 646

Lou Y.-Q., Fan Z. H., 2000b, in The Interstellar Medium in M31 and M33. 232 WE-Heraeus Seminar, eds. E.M. Berkhuijsen, R. Beck, R.A.M. Walterbos (Aachen: Shaker Verlag), pp. 205-208

Lou Y.-Q., Fan Z. H., 2002, MNRAS, 329, L62

Lou Y.-Q., Han J. L., Fan Z. H., 1999, MNRAS, 308, L1

Lou Y.-Q., Yuan C., Fan Z. H., 2001, ApJ, 552, 189

Lou Y.-Q., Yuan C., Fan Z. H., Leon S., 2001, ApJ, 553, L35

Lou Y.-Q., Walsh W. M., Han J. L., Fan Z. H., 2002, ApJ, 567, 289

Lu N. Y., et al., 1996, A\&A, 315, L153

Lynden-Bell D., 1966, Observatory, 86, 57

Malhotra S., et al., 1996, A\&A, 315, L161

Mathewson D. S., van der Kruit P. C., Brouw W. N., 1972, A\&A, 17, 468

Mestel L., 1963, MNRAS, 126, 553

Miller R. H., Prendergast K. H., Quirk W. J., 1970, ApJ, 161, 903

Montenegro L. E., Yuan C., Elmegreen B. G., 1999, ApJ, 520, 592

Mouschovias T. Ch., 1996, in Solar and Astrophysical Magnetohydrodynamic Flows, NATO ASI series, ed. Tsinganos, K. C. pp. 475-504, Kluwer Academic, Dordrecht.

Neininger N., 1992, A\&A, 263, 30

Neininger N., Beck R., Sukumar S., Allen R. J., 1993, A\&A, 274, 687

Ondrechen M. P., 1985, AJ, 90, 1474

Ostriker J. P., Peebles P. J. E., 1973, ApJ, 186, 467

Pacholczyk A. G., 1970, Radio Astrophysics, Freeman and Company, San Francisco, California

Pannatoni R. F., 1983, Geophys. Astrophys. Fluid Dyn., 24, 165

Parker E. N., 1965, ApJ, 142, 1086

Parker E. N., 1966, ApJ, 145, 811

Parker E. N., 1967 in Nebulae and Interstellar Matter (ed. by L. Aller, D. B. McLaughlin and B. Middlehurst) University of Chicago Press, Chicago, Vol. VII, Chapter 14 Dynamic Properties of Cosmic Rays. p. 707.

Parker E. N. 1969, Space Sci. Rev., 9, 651

Parker E. N., 1979, Cosmical Magnetic Fields. Clarendon, Oxford

Parker E. N., 1992, ApJ, 401, 137

Quirk W. J., 1972, ApJ, 176, L9

Rix H.-W., Rieke M. J., 1993, ApJ, 418, 123 
Roberts W. W., Jr., Yuan C., 1970, ApJ, 161, 887

Safronov V. S., 1960, Ann. d'Astrophysique, 23, 979

Scarrott S. M., Ward-Thompson D., Warren-Smith R. F., 1987, MNRAS, 224, 299

Shu F. H. 1970a, ApJ, 160, 89

Shu F. H. 1970b, ApJ, 160, 99

Shu, F. H. 1974, A\&A, 33, 55

Shu F. H., Laughlin G., Lizano S., Galli D., ApJ, 2000, 535, 190

Silk J., 1997, ApJ, 481, 703

Sofue Y., 1996, ApJ, 458, 120

Sukumar S., Allen R. J., 1989, Nature, 340, 537

Terquem C., Papaloizou J. C. B., 1996, MNRAS, 279, 767

Toomre A., 1964, ApJ, 139, 1217

Toomre A., 1977, ARA\&A, 15, 437

Toomre A., Toomre J., 1972, ApJ, 178, 623

Tuffs R. J., et al., 1996, A\&A, 315, L149

Velikhov E. P., 1959, J. Expl. Theoret. Phys. (U.S.S.R.), 36, 1398

Wang B., Silk J., 1994, ApJ, 427, 759

Woltjer L., 1965, in Galactic Structure, eds. Blaauw A., Schmidt M., pp. 531-587, The University of Chicago Press, Chicago

Wunderlich E., Klein U., Wielebinski R., 1987, A\&A, 69, 487

\section{APPENDIX A}

By requiring $u_{r}=v_{r}$, the lengthy equation (3.12) for $v_{r}$ contains the information of fast and slow MHD density waves and the suprathermal mode (Parker 1965) and can be cast into the compact mathematical form of

$$
\mathcal{C}_{1} \frac{d^{2} v_{r}}{d r^{2}}+\mathcal{C}_{2} \frac{d v_{r}}{d r}+\mathcal{C}_{3} v_{r}=0
$$

where the three complex coefficients $\mathcal{C}_{1}, \mathcal{C}_{2}$, and $\mathcal{C}_{3}$ are defined by

$$
\mathcal{C}_{1} \equiv \frac{i C_{A}^{2}}{(\omega-m \Omega)}+\frac{C_{S}^{2} \mathcal{B}}{\left(\mu_{\circ}+\epsilon_{\circ}\right)}+\frac{i(\omega-m \Omega) C_{C}^{2} \Sigma_{\circ}}{\left(\mu_{\circ}+\epsilon_{\circ}\right)\left[(\omega-m \Omega)^{2}-m^{2} C_{C}^{2} \Sigma_{\circ} /\left(r^{2} \epsilon_{\circ}\right)\right]},
$$




$$
\begin{aligned}
& \mathcal{C}_{2} \equiv-\frac{2 \Omega \mu_{\circ}}{\left(\mu_{\circ}+\epsilon_{\circ}\right)}\left[\frac{r(\omega-m \Omega) \mathcal{B}}{m \mu_{\circ}}-\frac{i r}{m}\right]-\frac{2 i m \Omega C_{C}^{2} \Sigma_{\circ}}{r\left(\mu_{\circ}+\epsilon_{\circ}\right)\left[(\omega-m \Omega)^{2}-m^{2} C_{C}^{2} \Sigma_{\circ} /\left(r^{2} \epsilon_{\circ}\right)\right]} \\
& +i C_{A}^{2}\left\{\frac{d}{d r}\left(\frac{1}{\omega-m \Omega}\right)+r \frac{d}{d r}\left[\frac{1}{r(\omega-m \Omega)}\right]\right\}-\mathcal{F}_{1} \mathcal{B}+\frac{1}{\left(\mu_{\circ}+\epsilon_{\circ}\right)} \frac{d\left(C_{S}^{2} \mathcal{B}\right)}{d r} \\
& +\frac{C_{C}^{2} \Sigma_{\circ}\left[i m \kappa^{2} /(2 \Omega r)+i(\omega-m \Omega) d \ln \left(r \Sigma_{\circ}\right) / d r\right]}{\left(\mu_{\circ}+\epsilon_{\circ}\right)\left[(\omega-m \Omega)^{2}-m^{2} C_{C}^{2} \Sigma_{\circ} /\left(r^{2} \epsilon_{\circ}\right)\right]}+\frac{C_{S}^{2} \mathcal{A}}{\left(\mu_{\circ}+\epsilon_{\circ}\right)} \\
& +\frac{1}{\left(\mu_{\circ}+\epsilon_{\circ}\right)} \frac{d}{d r}\left[\frac{C_{C}^{2} \Sigma_{\circ} i(\omega-m \Omega)}{(\omega-m \Omega)^{2}-m^{2} C_{C}^{2} \Sigma_{\circ} /\left(r^{2} \epsilon_{\circ}\right)}\right]-\frac{\mathcal{B}}{\left(\mu_{\circ}+\epsilon_{\circ}\right)^{2}} \frac{d\left(p_{\circ}+P_{\circ}\right)}{d r} \\
& -\frac{\Gamma C_{C}^{2} \Sigma_{\circ} /\left[(\Gamma-1) c^{2}\right]}{\left(\mu_{\circ}+\epsilon_{\circ}\right)^{2}} \frac{d\left(p_{\circ}+P_{\circ}\right)}{d r} \frac{i(\omega-m \Omega)}{(\omega-m \Omega)^{2}-m^{2} C_{C}^{2} \Sigma_{\circ} /\left(r^{2} \epsilon_{\circ}\right)} \text {, } \\
& \mathcal{C}_{3} \equiv i(\omega-m \Omega)-\frac{2 \Omega \mu_{\circ}}{\left(\mu_{\circ}+\epsilon_{\circ}\right)}\left[\frac{r(\omega-m \Omega)}{m \mu_{\circ}} \mathcal{A}-\frac{i}{m \mu_{\circ}} \frac{d\left(r \mu_{\circ}\right)}{d r}\right] \\
& -\frac{2 \Omega \epsilon_{\circ}}{\left(\mu_{\circ}+\epsilon_{\circ}\right)}\left[\frac{m C_{C}^{2} \Sigma_{\circ}}{r(\omega-m \Omega) \epsilon_{\circ}} \frac{i m \kappa^{2} /(2 \Omega r)+i(\omega-m \Omega) d \ln \left(r \Sigma_{\circ}\right) / d r}{(\omega-m \Omega)^{2}-m^{2} C_{C}^{2} \Sigma_{\circ} /\left(r^{2} \epsilon_{\circ}\right)}\right. \\
& \left.+\frac{i \kappa^{2}}{2 \Omega(\omega-m \Omega)}\right]-\frac{i m^{2} C_{A}^{2}}{r^{2}(\omega-m \Omega)}+i C_{A}^{2} \frac{d}{d r}\left\{r \frac{d}{d r}\left[\frac{1}{r(\omega-m \Omega)}\right]\right\} \\
& -\mathcal{F}_{1} \mathcal{A}+\frac{1}{\left(\mu_{\circ}+\epsilon_{\circ}\right)} \frac{d\left(C_{S}^{2} \mathcal{A}\right)}{d r}-\frac{\mathcal{A}}{\left(\mu_{\circ}+\epsilon_{\circ}\right)^{2}} \frac{d\left(p_{\circ}+P_{\circ}\right)}{d r} \\
& +\frac{1}{\left(\mu_{\circ}+\epsilon_{\circ}\right)} \frac{d}{d r}\left\{\frac{C_{C}^{2} \Sigma_{\circ}\left[i m \kappa^{2} /(2 \Omega r)+i(\omega-m \Omega) d \ln \left(r \Sigma_{\circ}\right) / d r\right]}{(\omega-m \Omega)^{2}-m^{2} C_{C}^{2} \Sigma_{\circ} /\left(r^{2} \epsilon_{\circ}\right)}\right\} \\
& -\frac{\Gamma C_{C}^{2} \Sigma_{\circ} /\left[(\Gamma-1) c^{2}\right]}{\left(\mu_{\circ}+\epsilon_{\circ}\right)^{2}} \frac{d\left(p_{\circ}+P_{\circ}\right)}{d r} \frac{\left[i m \kappa^{2} /(2 \Omega r)+i(\omega-m \Omega) d \ln \left(r \Sigma_{\circ}\right) / d r\right]}{(\omega-m \Omega)^{2}-m^{2} C_{C}^{2} \Sigma_{\circ} /\left(r^{2} \epsilon_{\circ}\right)} \text {. }
\end{aligned}
$$

The complex coefficients $\mathcal{F}_{1}, \mathcal{A}$, and $\mathcal{B}$ are defined by equations (3.5) and (3.6) in the main text.

\section{APPENDIX B}

For the convenience of reference and discussion, we derive and summarize here several pertinent phase relationships among perturbation variables, with a generalization of the original analysis by Parker (1965) for hydromagnetic perturbations in a uniformly magnetized medium of thermal and suprathermal gases. To accommodate the possible parameter regime of $W \equiv B /\left[4 \pi\left(\rho_{\circ}+\delta_{\circ}\right)\right]^{1 / 2} \gtrsim c$ with $c$ being the speed of light, we include the effect of displacement current perturbation for the Lorentz force term in the momentum equation. Otherwise, the Cartesian coordinates $(x, y, z)$, notations, and definitions all remain essentially the same as those adopted by Parker (1965).

From the mass conservation and $z$-component of the momentum equation for the thermal gas, one has

$$
u_{y}=\frac{\rho\left(\omega^{2}-k^{2} a^{2} \cos ^{2} \theta\right)}{\omega \rho_{\circ} k \sin \theta},
$$


where $u_{y}$ is the bulk velocity perturbation of the thermal gas perpendicular to the background magnetic field $B \hat{z}, \omega$ is the angular frequency, $k$ is the total wavenumber, $\theta$ is the angle in the $y z$-plane between the wave vector $\vec{k}$ and the background magnetic field $B \hat{z}, a$ is the sound speed of the thermal gas, $\rho_{\circ}$ is the uniform background thermal gas density, and $\rho$ is the thermal gas density perturbation. In parallel, from the mass conservation and $z$-component of the momentum equation for the suprathermal gas, one has

$$
v_{y}=\frac{\delta\left(\omega^{2}-k^{2} b^{2} \cos ^{2} \theta\right)}{\omega \delta_{\circ} k \sin \theta},
$$

where $v_{y}$ is the bulk velocity perturbation of the suprathermal gas perpendicular to the background magnetic field $B \hat{z}, b$ is the sound speed in the suprathermal gas, $\delta_{\circ}$ is the uniform background suprathermal gas density, and $\delta$ is the suprathermal gas density perturbation. By the requirement that the bulk velocities of thermal gas and superathermal gas are the same transverse to the background magnetic field $B \hat{z}$, namely $u_{y}=v_{y}$, we derive from equations $(B 1)$ and $(B 2)$

$$
\frac{\delta}{\delta_{\circ}}=\frac{\rho\left(\omega^{2}-k^{2} a^{2} \cos ^{2} \theta\right)}{\rho_{\circ}\left(\omega^{2}-k^{2} b^{2} \cos ^{2} \theta\right)},
$$

where $\delta / \delta_{\circ}$ and $\rho / \rho_{\circ}$ are the relative mass density fluctuations in the suprathermal and thermal gases, respectively.

By combining the $z$-component of the magnetic induction equation

$$
\omega b_{z}=B k \sin \theta u_{y}
$$

with equation $(B 1)$ where $b_{z}$ is the $z$-component of the magnetic field perturbation, one has the relative fluctuation in parallel magnetic field $b_{z} / B$ and the relative mass density fluctuation in thermal gas $\rho / \rho_{\text {。 }}$ related by

$$
\frac{b_{z}}{B}=\frac{\rho\left(\omega^{2}-k^{2} a^{2} \cos ^{2} \theta\right)}{\rho_{\circ} \omega^{2}} .
$$

The incompressible transverse Alfvén mode involves $u_{x}=v_{x}$ and $b_{x} \neq 0$, and solution (9) of Parker (1965) for its dispersion relation now takes the form of

$$
\omega^{2}=\frac{k^{2} W^{2} \cos ^{2} \theta}{\left(1+W^{2} / c^{2}\right)},
$$

where $W \equiv B /\left[4 \pi\left(\rho_{\circ}+\delta_{\circ}\right)\right]^{1 / 2}$. The modified dimensionless dispersion relation for the fast, slow, and suprathermal modes now becomes

$$
\begin{aligned}
\left(U^{2}-M^{2}\right) & \left(U^{2}-\cos ^{2} \theta\right)\left(U^{2}-n^{2} \cos ^{2} \theta\right) \\
& -\frac{U^{2} \sin ^{2} \theta\left[(1+\alpha) U^{2}-\left(1+\alpha / n^{2}\right) n^{2} \cos ^{2} \theta\right]}{\left(1+\alpha / n^{2}\right)\left(1+W^{2} / c^{2}\right)}=0,
\end{aligned}
$$

where $U \equiv \omega /(k a), M^{2} \equiv W^{2} /\left[a^{2}\left(1+W^{2} / c^{2}\right)\right], n \equiv b / a, \alpha \equiv \delta_{\circ} b^{2} /\left(\rho_{\circ} a^{2}\right)$ (for a comparison, see Parker's eqn [15] and the relevant definitions). The factor $\left(1+W^{2} / c^{2}\right)$ that appears in various places is due to the displacement current 
effect. We now examine systematically the phase relationships among various perturbation enhancements such as $\rho / \rho_{\circ}, b_{z} / B$, and $\delta / \delta_{\circ}$ for the fast, slow, and suprathermal modes separately, using the phase relations $(B 3)$ and $(B 5)$.

1. The special case of $\theta=0$ (parallel wave propagations relative to $\vec{B}$ ).

Dispersion relation $(B 7)$ gives three exact solutions,

$$
\begin{gathered}
U^{2}=M^{2}, \\
U^{2}=1, \\
U^{2}=n^{2} .
\end{gathered}
$$

For the modified Alfvén wave speed $C_{D} \equiv W /\left(1+W^{2} / c^{2}\right)^{1 / 2}$ greater (or less) than the thermal sound speed $a$, solution $(B 8)$ corresponds to the fast (or slow) mode, while solution ( $B 9)$ corresponds to the slow (or fast) mode. Solution $(B 10)$ represents the suprathermal mode.

For the suprathermal mode $(B 10)$ with $\omega^{2}=k^{2} b^{2}$, one gets $\rho=0, p=0$, $u_{z}=0, u_{y}=0, \delta \neq 0, P \neq 0, v_{z} \neq 0, b_{y}=0$, and $b_{z}=0$. Physically, this is an acoustic wave in the suprathermal gas propagating along the background magnetic field $\vec{B}$ without disturbing the thermal gas. For a relativistically hot $\mathrm{CRG}$, the value of $b$ can be fairly close to the speed of light $c$.

For $C_{D}>a$, the slow mode $(B 9)$ with $\omega^{2}=k^{2} a^{2}$ gives $\rho \neq 0, p \neq 0, u_{z} \neq 0$, $u_{y}=0, \delta=0, P=0, v_{z}=0, b_{y}=0$, and $b_{z}=0$. Physically, this is an acoustic wave in the thermal gas propagating along the background magnetic field $\vec{B}$ without disturbing the suprathermal gas.

For $C_{D}>a$, the fast mode $(B 8)$ with $\omega^{2}=k^{2} W^{2}$ involves all nonzero perturbation variables. By equation $(B 3), \rho / \rho_{\circ}$ and $\delta / \delta_{\circ}$ are out of phase, while by equation $(B 5), \rho / \rho_{\circ}$ and $b_{z} / B$ are in phase as expected.

For $C_{D}<a$, the fast mode $(B 9)$ with $\omega^{2}=k^{2} a^{2}$ has the same characteristics of an acoustic wave in the thermal gas propagating along the background magnetic field. One simply changes the name of this mode because of a faster wave speed.

For $C_{D}<a$, the slow mode $(B 8)$ with $\omega^{2}=k^{2} W^{2}$ involves all nonzero perturbation variables. By equation $(B 3), \rho / \rho_{\circ}$ and $\delta / \delta_{\circ}$ are now in phase, while by equation $(B 5), \rho / \rho_{\circ}$ and $b_{z} / B$ are now out of phase as expected.

2. The case of $\theta \sim \pi / 2$ (nearly perpendicular wave propagations relative to $\vec{B}$ ).

Here, we would first correct typos in Parker's paper in the case of $\theta$ being sufficiently close to $\pi / 2$ : Parker's solution (19)

$$
U^{2}=\frac{M^{2}}{M^{2}+1} \cos ^{2} \theta
$$

should be associated with the minus (not plus) sign of $R$ for the slow mode, while his solution $(20)$

$$
U^{2} \cong n^{2} \cos ^{2} \theta \frac{M^{2}+1}{M^{2}+1+\alpha}
$$


should be associated with the plus (not minus) sign of $R$ for the fast mode, where $R$ is explicitly defined by his equation (18), namely

$$
R \equiv \pm\left[\left(M^{2}+1\right)^{2}-4 M^{2} \cos ^{2} \theta\right]^{1 / 2}
$$

(see his eqn. [15] or our eqn. [B7] with $W^{2} / c^{2} \ll 1$ ). Also, a more accurate version of his solution (21) for the suprathermal mode close to $\theta=\pi / 2$ would be

$$
U^{2} \cong M^{2}+\frac{\alpha+1}{1+\alpha / n^{2}}+n^{2} \cos ^{2} \theta \frac{\alpha}{M^{2}+\alpha+1}
$$

as can be readily seen from his equation (15) by setting $\theta=\pi / 2$.

In the presence of displacement current effects, one can also derive three approximate solutions from dispersion relation $(B 7)$ that may be written in the polynomial form of

$$
\begin{aligned}
& U^{6}-\left[\left(n^{2}+1\right) \cos ^{2} \theta+M^{2}+\frac{(1+\alpha) \sin ^{2} \theta}{\left(1+\alpha / n^{2}\right)\left(1+W^{2} / c^{2}\right)}\right] U^{4} \\
& +\cos ^{2} \theta\left[\left(\cos ^{2} \theta+\frac{\sin ^{2} \theta}{1+W^{2} / c^{2}}\right) n^{2}+M^{2}\left(n^{2}+1\right)\right] U^{2}-M^{2} n^{2} \cos ^{4} \theta=0,
\end{aligned}
$$

where $M^{2} \equiv W^{2} /\left[a^{2}\left(1+W^{2} / c^{2}\right)\right]$. For $\theta \sim \pi / 2$ and $n^{2} \gg 1$, the dispersion relation of the slow mode now becomes

$$
U^{2} \cong \frac{M^{2} \cos ^{2} \theta}{M^{2}\left(1+1 / n^{2}\right)+\sin ^{2} \theta /\left(1+W^{2} / c^{2}\right)+\cos ^{2} \theta},
$$

the dispersion relation of the fast mode now becomes

$$
U^{2} \cong \frac{\cos ^{2} \theta\left\{\left[\cos ^{2} \theta+\sin ^{2} \theta /\left(1+W^{2} / c^{2}\right)\right] n^{2}+M^{2}\left(n^{2}+1\right)\right\}}{\left(n^{2}+1\right) \cos ^{2} \theta+M^{2}+(1+\alpha) \sin ^{2} \theta /\left[\left(1+\alpha / n^{2}\right)\left(1+W^{2} / c^{2}\right)\right]},
$$

and the dispersion relation of the suprathermal mode now becomes

$$
U^{2} \cong M^{2}+\frac{\alpha+1}{\left(1+\alpha / n^{2}\right)\left(1+W^{2} / c^{2}\right)}+\frac{\alpha n^{2} \cos ^{2} \theta}{M^{2}+\alpha+1} .
$$

For the slow mode $(B 16), b_{z} / B$ and $\rho / \rho_{\circ}$ are out of phase by using equation $(B 5)$ as expected, while $\delta / \delta_{\circ}$ and $\rho / \rho_{\circ}$ are in phase by using equation $(B 3)$.

For the fast mode $(B 17), b_{z} / B$ and $\rho / \rho_{\circ}$ are in phase by using equation $(B 5)$, while $\delta / \delta_{\circ}$ and $\rho / \rho_{\circ}$ are out of phase by using equation $(B 3)$.

For the suprathermal mode $(B 18), b_{z} / B$ and $\rho / \rho_{\circ}$ are in phase by using equation $(B 5)$, and $\delta / \delta_{\circ}$ and $\rho / \rho_{\circ}$ are also in phase by using equation $(B 3)$.

3. The case of $n^{2} \cos ^{2} \theta$ being sufficiently larger than 1 with $\theta \neq 0$. have

Corresponding to the suprathermal mode of Parker's solution (17), we now

$$
U^{2}=n^{2} \cos ^{2} \theta+\frac{\alpha \sin ^{2} \theta}{1+W^{2} / c^{2}}+\mathcal{O}\left(\frac{1}{n^{2} \cos ^{2} \theta}\right)
$$


and one can readily show that $\delta / \delta_{\circ}$ and $\rho / \rho_{\circ}$ are in phase by equation $(B 3)$, and $b_{z} / B$ and $\rho / \rho_{\circ}$ are also in phase by equation $(B 5)$.

Corresponding to the fast and slow modes of Parker's solution (16), we now have

$$
\begin{aligned}
& U^{2}= \frac{\left[M^{2}+\cos ^{2} \theta+\sin ^{2} \theta /\left(1+W^{2} / c^{2}\right)+R_{D}\right]}{2} \\
& \times\left\{1-\frac{\alpha\left[M^{2}+\cos ^{2} \theta+\sin ^{2} \theta /\left(1+W^{2} / c^{2}\right)+R_{D}\right] \tan ^{2} \theta}{2 R_{D} n^{2}\left(1+W^{2} / c^{2}\right)}\right\} \\
&+\mathcal{O}\left(\frac{1}{n^{4} \cos ^{4} \theta}\right)
\end{aligned}
$$

where, replacing definition $(B 13)$ for $R, R_{D}$ is defined by

$$
R_{D} \equiv \pm\left\{\left[M^{2}+\cos ^{2} \theta+\sin ^{2} \theta /\left(1+W^{2} / c^{2}\right)\right]^{2}-4 M^{2} \cos ^{2} \theta\right\}^{1 / 2}
$$

with the plus and minus signs of $R_{D}$ corresponding to the fast and slow modes, respectively.

For the fast mode $(B 20)$ with the plus sign of $R_{D}$ in definition $(B 21)$, one can show that $\delta / \delta_{\circ}$ and $\rho / \rho_{\circ}$ are out of phase by equation $(B 3)$, while $b_{z} / B$ and $\rho / \rho_{\circ}$ are in phase by equation (B5) as expected.

Meanwhile, for the slow mode $(B 20)$ with the minus sign of $R_{D}$ in definition $(B 21)$, one can show that $\delta / \delta_{\circ}$ and $\rho / \rho_{\circ}$ are in phase by equation (B3), while $b_{z} / B$ and $\rho / \rho_{\circ}$ are out of phase by equation (B5) as expected.

We now summarize the results for the parameter regime of $n \gg 1$ (i.e., $b \gg a$ ) with comparable $W$ and $a$.

1. For the suprathermal mode, $\delta / \delta_{\circ}, \rho / \rho_{\circ}$, and $b_{z} / B$ are all in phase.

2. For the fast mode, $\delta / \delta_{\circ}$ and $b_{z} / B$ are out of phase with $\rho / \rho_{\circ}$ and $b_{z} / B$ being in phase.

3. For the slow mode, $\delta / \delta_{\circ}$ and $b_{z} / B$ are out of phase with $\rho / \rho_{\circ}$ and $b_{z} / B$ being out of phase.

\section{APPENDIX C}

The mass conservation for the interstellar medium (ISM) is

$$
\frac{\partial \rho}{\partial t}+\nabla \cdot(\rho \vec{v})=0
$$

where $\rho$ is the ISM mass density and $\vec{v}$ is the bulk flow velocity of the ISM. After the operation of vertical integration $\int d z$, one has

$$
\frac{\partial \mu}{\partial t}+\nabla \cdot(\mu \vec{v})=0
$$

where $\mu \equiv \int d z \rho$ is the surface mass density of the ISM. 
Similarly, the mass conservation for the cosmic-ray gas (CRG) is

$$
\frac{\partial \delta}{\partial t}+\nabla \cdot(\delta \vec{u})=0
$$

where $\delta$ is the mass density and $\vec{u}$ is the bulk flow velocity of the CRG. After the operation of vertical integration $\int d z$, one has

$$
\frac{\partial \Sigma}{\partial t}+\nabla \cdot(\Sigma \vec{u})=0
$$

where $\Sigma \equiv \int d z \delta$ is the surface mass density of the CRG.

We invoke the polytropic approximations for both the ISM and the CRG,

$$
p=C_{S}^{2} \mu
$$

and

$$
P=C_{C}^{2} \Sigma
$$

where $C_{S}$ is the thermal sound speed of the ISM and $C_{C}$ is the effective sound speed of the CRG, $p$ is the vertically integrated (two-dimensional) gas pressure of the ISM, and $P$ is the vertically integrated (two-dimensional) effective pressure of the CRG which is regarded as a "relativistically hot" gas.

For a plasma of infinite conductivity, the magnetic induction equation is

$$
\frac{\partial \vec{B}}{\partial t}=\nabla \times(\vec{v} \times \vec{B})
$$

where the magnetic field $\vec{B}$ satisfies the divergence-free condition of

$$
\nabla \cdot \vec{B}=0
$$

In the current context, bulk flow velocities $\vec{v}_{\perp}$ and $\vec{u}_{\perp}$ perpendicular to $\vec{B}$ are constrained to be the same by the large-scale mean magnetic field.

By ignoring effects of displacement current for nonrelativistic flows, we have

$$
\nabla \times \vec{B}=\frac{4 \pi}{c}\left(\vec{J}_{I S M}+\vec{J}_{C R G}\right)
$$

where $c$ is the speed of light, $\vec{J}_{I S M}$ and $\vec{J}_{C R G}$ are the electric current densities present in the ISM and in the CRG, respectively.

The (two-dimensional) momentum equation for the ISM is

$$
\mu \frac{D \vec{v}}{D t}+\nabla p-\int d z \frac{\vec{J}_{I S M} \times \vec{B}}{c}-\mu \nabla \Phi=0
$$

and the (two-dimensional) momentum equation for the CRG is

$$
\Sigma \frac{D}{D t}\left(\frac{\psi \vec{u}}{\bar{m} c^{2}}\right)+\nabla P-\int d z \frac{\vec{J}_{C R G} \times \vec{B}}{c}-\frac{\Sigma \psi}{\bar{m} c^{2}} \nabla \Phi=0,
$$

where $\bar{m}$ is the mean particle mass in the CRG, $\Phi$ is the negative gravitational potential, $\psi \equiv \bar{m} c^{2}+\Gamma \bar{m} P /[(\Gamma-1) \Sigma]$ is the proper specific enthalpy including that of the particle rest mass. Given the polytropic approximation, $\psi$ is a constant.

For the background rotational equilibrium in the radial direction, the above two equations can be combined to derive equation (2.1) (denoting $\epsilon \equiv \Sigma \psi /\left[\bar{m} c^{2}\right]$ ). Likewise, perturbations of these two equations can be combined to derive the radial component equation (2.6) by using equation (2.1) and the force-free approximation. 